\title{
Displacement-Reduction Routes to PtPd Clusters and Mechanistic Inferences for the Synthesis of Other Bimetallic Compositions
}

\author{
Alexandra M. Landry and Enrique Iglesia* \\ Department of Chemical and Biomolecular Engineering, University of California, Berkeley, CA 94720, USA \\ Chemical Sciences Division, E.O. Lawrence Berkeley National Laboratory, Berkeley, CA 94720, USA \\ *E-mail: iglesia@berkeley.edu
}

(C) 2016. This manuscript version is made available under the Elsevier user license 


\begin{abstract}
Bimetallic PtPd clusters (2.1-2.9 nm) dispersed on $\mathrm{SiO}_{2}$ and uniform in composition and size were prepared using colloidal methods with reagents containing only $\mathrm{C}, \mathrm{O}, \mathrm{H}$, and $\mathrm{N}$ atoms. These synthetic protocols extend galvanic displacement-reduction (GDR) processes previously used to prepare AuPd and AuPt clusters. Such processes exploit the different redox potentials of two elements to encourage their deposition within the same cluster. The size, composition, and formation mechanism of PtPd clusters were probed using transmission electron microscopy, UVvisible spectroscopy, energy-dispersive X-ray spectroscopy, and high-angle annular dark-field imaging. Taken together with previous data for AuPd and AuPt systems, these findings highlight key general features, properties, and protocols required to form uniform bimetallic clusters. Exothermic alloys, such as PtPd and AuPd, form predominantly via selective GDR routes; in contrast, alternate routes become significant for endothermic alloys, such as AuPt. Bimetallic clusters grow via GDR processes ( $\mathrm{PtPd}, \mathrm{AuPd})$ at rates proportional to the surface area of each cluster; therefore, compositional uniformity is dictated by the size distribution of the seed clusters. The rate of GDR processes reflects the difference in reduction potentials of the two components, as shown by more facile formation of AuPd than PtPd clusters. These considerations and experimental evidence provide useful guidance for conditions and protocols likely to succeed for other bimetallic pairs. Low-temperature $(\leq 423 \mathrm{~K})$ reductive treatments (in $\mathrm{H}_{2}$ or EtOH) successfully removed all synthetic detritus from Pd and PtPd clusters dispersed on $\mathrm{SiO}_{2}$, without significant coalescence. Such removal strategies are more challenging for Pd than for Pt clusters because of stronger Pd-polymer bonds and the greater sintering tendency of Pd clusters.
\end{abstract}

Keywords: colloidal synthesis; bimetallic catalyst; galvanic displacement; uniform nanoparticles; platinum; gold; palladium; polymer removal; polyvinylpyrrolidone 


\section{Introduction}

Bimetallic catalysts have been shown to increase catalytic turnover rates, improve selectivity, and enhance stability [1,2]. The reported interpretations of the effects of alloying on the reactivity of small bimetallic clusters are often equivocal or inaccurate, however, because of the complexity introduced by the size and compositional heterogeneity of bimetallic materials prepared by typical synthesis techniques, such as incipient wetness impregnation [3-6]. Alternatively, electroless deposition (ED) [7-10] and galvanic displacement (GD) [11, 12] techniques selectively reduce solvated metal cations onto the surfaces of supported metal clusters by activating the metal surface with a reducing agent or using the metal surface itself as the reducing agent, respectively; such methods can exclusively form bimetallic particles. The selective placement of the second metal, however, is impeded upon obstruction of the seed cluster surface with the deposited metal, thus limiting the bimetallic compositions that can be prepared. Metal cation-support interactions must also be carefully avoided in order to prevent the formation of monometallic particles on the support, and the broad particle size distributions of the initial supported metal clusters prepared by incipient wetness impregnation lead to broad bimetallic particle size distributions. Colloidal methods, however, avoid all metal-support interactions during cluster formation, while also providing better control over particle composition and size [2, 13]; in spite of this, they frequently require the use of reagents that poison the catalyst surface (e.g., protecting agents with thiol groups) and thus influence turnover rates. Here, we describe the colloidal synthesis of PtPd nanoparticles (2.1-2.9 $\mathrm{nm}$ ) uniform in composition and size via galvanic displacement-reduction (GDR) methods using reagents comprised of only $\mathrm{C}, \mathrm{O}, \mathrm{H}$, and $\mathrm{N}$ atoms; we show that such materials can be removed from $\mathrm{SiO}_{2}$-supported $\mathrm{Pd}$ and $\mathrm{PtPd}$ cluster surfaces without significant particle agglomeration using low-temperature reductive treatments, thus yielding chemically accessible metal catalyst surfaces. We discuss our results within the broader context of developing bimetallic procedures for other metal combinations based on comparisons of PtPd with AuPt [14] and AuPd [15] bimetallic systems prepared using similar methods.

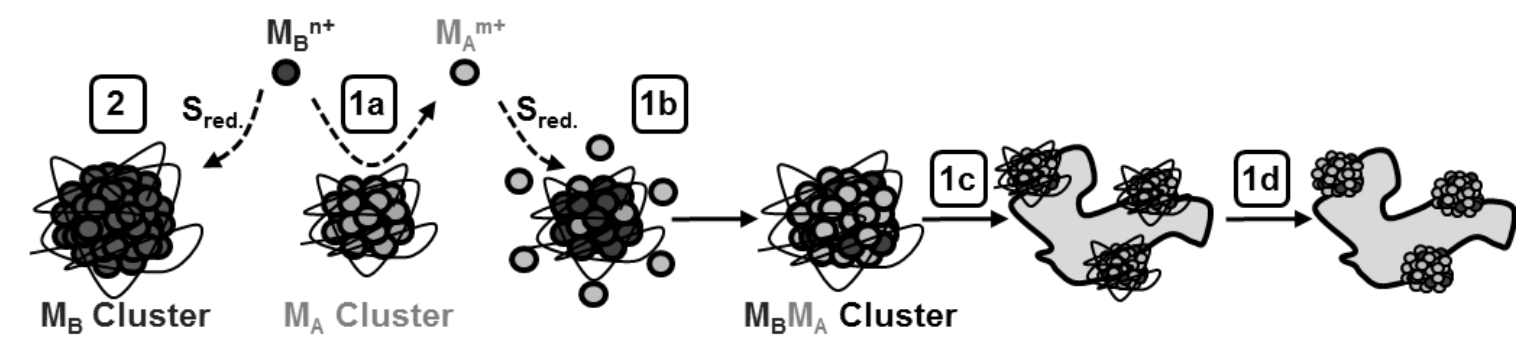

Homo- or Heterogeneous Nucleation

Galvanic Displacement-Reduction

Catalyst Preparation

Scheme 1. Protocols for the colloidal synthesis of bimetallic catalysts via galvanic displacementreduction. 1a) Metal surface atoms of polymer-protected seed particles $\left(\mathbf{O}, \mathbf{M}_{\mathrm{A}}\right.$; black lines surrounding cluster represent the polymer) reduce solvated metal cations $\left(\mathbf{O}, \mathrm{M}_{\mathrm{B}}\right)$ of the more noble metal, driven by a positive $\Delta \mathrm{E}_{\text {redox }}^{\mathrm{o}}\left(\mathrm{E}_{\mathrm{MB}}^{\mathrm{o}}>\mathrm{E}_{\mathrm{MA}}^{\mathrm{o}}\right)$. 1b) The solvated $\mathrm{M}_{\mathrm{A}}$ cations formed from the redox reaction in step 1 a subsequently get reduced by the solvent, a reductant $\left(S_{\text {red. }}\right)$, back onto the bimetallic particle surface. 1c) Clusters are deposited onto the support and 1d) the protecting polymer is removed. The preparation of uniform bimetallic particles requires preventing the nucleation of $\mathrm{M}_{\mathrm{B}}$ (path 2). Scheme modified from previously reported work [14]. 
PtPd clusters were synthesized in this study using galvanic displacement-reduction (GDR) protocols previously shown to form AuPt [14] and AuPd [15] bimetallic nanoparticles. The synthesis of AuPt and AuPd clusters begins with a colloidal suspension of nanoparticles composed of the less noble metal $\left(\mathrm{M}_{\mathrm{A}}=\mathrm{Pt}\right.$ or $\left.\mathrm{Pd}\right)$, which is subsequently combined with metal cations of the more noble species $\left(\mathrm{M}_{B}=A u\right)$. The reduction of solvated $\mathrm{M}_{B}$ cations by surface $\mathrm{M}_{\mathrm{A}}$ atoms is thermodynamically favorable, as indicated by their relative standard reduction potentials $\left(\mathrm{E}^{\mathrm{o}}, \Delta \mathrm{E}_{\text {redox }}^{\mathrm{o}}=\mathrm{E}_{\mathrm{B}}^{\mathrm{O}}-\mathrm{E}_{\mathrm{A}}^{\mathrm{o}}>0\right.$; Table 1), thus leading to the reduction and deposition of $\mathrm{M}_{\mathrm{B}}$ atoms onto cluster surfaces and to the oxidation and dissolution of $\mathrm{M}_{\mathrm{A}}$ atoms into the solvent (Scheme 1.1a). The solvent, also acting as the reductant (ethanol or ethylene glycol for AuPd or AuPt clusters, respectively), is at a much higher concentration than the counterions of the metal precursors (e.g. $\mathrm{NO}_{3}{ }^{-}$or $\mathrm{Cl}^{-}$), and thus coordinate to the $\mathrm{M}_{\mathrm{A}}$ cations and subsequently reduce and deposit them back onto particle surfaces (Scheme 1.1b) at greater rates than the reduction of the $\mathrm{M}_{\mathrm{B}}$ metal precursors (e.g. $\mathrm{HAuCl}_{4}$ ) [16]. Consequently, the kinetically relevant step of the GDR sequence is the galvanic displacement step (Scheme 1.1a) [14, 15]. Re-reduction of $M_{A}$ atoms by the solvent ensures that $\mathrm{M}_{\mathrm{A}}$ atoms are continuously accessible at the particle surface to reduce $\mathrm{M}_{\mathrm{B}}$ cations; the presence of $\mathrm{M}_{\mathrm{A}}$ atoms (Pt or Pd) at the AuPd or AuPt bimetallic particle surface was confirmed by UV-visible spectroscopy.

These mechanistic details led us to conclude that the GDR approach would also lead to successful syntheses of other metal combinations, provided that cations of the nobler metal are added to suspensions of clusters composed of the less noble metal in the presence of a reducing agent. We show here that this is indeed the case for PtPd clusters, which were synthesized by adding a Pt precursor solution to a pre-formed suspension of Pd seed clusters in an ethanol-water mixture, a sequence prescribed by guidance from their respective reduction potentials $\left(\Delta \mathrm{E}_{\text {redox }}^{\mathrm{o}}=\right.$ $\mathrm{E}_{\mathrm{Pt}}^{\mathrm{O}}-\mathrm{E}_{\mathrm{Pd}}^{\mathrm{o}}>0$; Table 1). In contrast with Au-containing systems, however, the absence of unique UV-visible spectral features to distinguish between Pt and Pd surfaces indicates that the ED mechanism may also occur in parallel with GDR during bimetallic cluster formation.

Table 1. [17] Standard reduction potentials of elements of catalytic interest.

\begin{tabular}{cc} 
& Standard Reduction \\
Metal & Potential $\left(\mathrm{E}^{\circ}, \mathrm{V}\right)$ \\
$\mathrm{Au}^{3+}$ & 1.52 \\
$\mathrm{Pt}^{2+}$ & 1.18 \\
$\mathrm{Pd}^{2+}$ & 0.99 \\
$\mathrm{Ir}^{3+}$ & 0.92 \\
$\mathrm{Ag}^{1+}$ & 0.80 \\
$\mathrm{Rh}^{2+}$ & 0.60 \\
$\mathrm{Ru}^{3+}$ & 0.38 \\
$\mathrm{Ni}^{2+}$ & -0.26 \\
$\mathrm{Co}^{2+}$ & -0.28 \\
\hline
\end{tabular}

PtPd particles were characterized using transmission electron microscopy, UV-visible spectroscopy, energy dispersive X-ray spectroscopy (EDS), and high-angle annular dark field (HAADF) imaging. Particle-by-particle EDS in tandem with HAADF imaging demonstrated the exclusive formation of bimetallic clusters; these data were also used to confirm the mechanistic 
features of GDR that are relevant to the compositional uniformity of these bimetallic catalysts. $\mathrm{M}_{\mathrm{A}}$ seed clusters that grow via GDR processes increase in size at rates proportional to their surface areas, thus increasing in diameter by a thickness $(\Delta d)$ that is independent of the initial seed particle size [15]. Consequently, a distribution of $M_{A}$ seed cluster sizes all growing by the same thickness $(\Delta d)$ of $\mathrm{M}_{\mathrm{B}}$ leads to a distribution of bimetallic compositions such that the atomic fraction of $\mathrm{Au}$ decreases with increasing parent seed cluster size [14]. The compositional uniformity of a bimetallic sample prepared by GDR methods is therefore dictated by the breadth of the size distribution of the parent seeds.

Results presented here for PtPd, in addition to those shown previously for AuPd [15] and AuPt [14] systems, indicate that the relative reduction potentials of the two alloyed metals, as well as their mixing enthalpies, play consequential roles in the formation of bimetallic clusters via GDR. The favorable mixing thermodynamics of miscible alloys such as AuPd and PtPd $\left(\Delta \mathrm{H}_{\mathrm{AuPd}}=-6.7 \mathrm{~kJ} \mathrm{~mol}^{-1}[18], \Delta \mathrm{H}_{\mathrm{PtPd}}=-4.3 \mathrm{~kJ} \mathrm{~mol}^{-1}\right.$ [19]) ensure that metal atoms remain mixed within clusters and therefore retain at cluster surfaces the $\mathrm{M}_{\mathrm{A}}$ atoms required for GDR. In the case of PtPd synthesis, however, a lower thermodynamic driving force for the galvanic displacement (Scheme 1.1a) of $\mathrm{Pd}^{0}$ seed surface atoms by $\mathrm{Pt}$ cations relative to $\mathrm{Au}$ cations

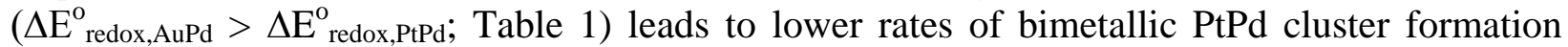
compared with those of AuPd formation. The endothermic nature of AuPt mixing $\left(\Delta \mathrm{H}_{\mathrm{AuPt}}=+2.3\right.$ $\mathrm{kJ} \mathrm{mol}^{-1}$ [20]) results in intraparticle segregation and to Au enrichment at cluster surfaces, thus inhibiting GDR by $\mathrm{Pt}^{0}$ sites and introducing additional growth pathways that lead to non-uniform distributions of $\mathrm{Au}$ atoms among the Pt seeds [14]. Solvents with high reduction potentials (e.g. butanediol), which compete with $\mathrm{M}_{\mathrm{A}}$ surface atoms as reductants of $\mathrm{M}_{\mathrm{B}}$ cations, have also been shown to lead to bimodal distributions of bimetallic clusters by promoting the homogeneous or heterogeneous nucleation of $\mathrm{M}_{\mathrm{B}}$-rich clusters. Employing GDR protocols to prepare bimetallic clusters of immiscible metals or to prepare clusters within solvents of high reduction potentials therefore requires more rigid synthesis protocols to promote the formation of uniform bimetallic clusters [14].

These systematic studies of PtPd, AuPd, and AuPt systems have demonstrated the general applicability of synthetic protocols that exploit GDR processes in preparing bimetallic clusters of diverse metal combinations using innocuous reagents that can be removed by low-temperature treatments; these studies have also shown how differences in $\Delta \mathrm{E}_{\text {redox }}^{\mathrm{r}}$, metal miscibility, and solvent reduction potential require, in turn, that such synthesis protocols be adapted to prevent the formation of bimodal particle distributions [14, 15]. In addition to the three bimetallic systems discussed herein, we propose that other catalyst compositions - such as PdRh starting from Rh seed clusters or PtAg starting from Ag seed clusters (Table 1) — can also be synthesized using the GDR approach.

\section{Materials and methods}

\subsection{Catalyst synthesis}

\subsubsection{Preparation of polyvinylpyrrolidone $(P V P)$ protected Pd clusters $(1.8 \mathrm{~nm})$}

A mixture of $1: 1$ by volume ethanol $(\mathrm{EtOH}$, Sigma Aldrich, $>99.5 \%)$ and deionized water $\left(\mathrm{H}_{2} \mathrm{O}\right.$; conductivity $\left.\sim 0.06 \mu \mathrm{S} \mathrm{cm}^{-1}\right)$ with solvated PVP $(0.02 \mathrm{M}$, monomeric unit basis; Sigma Aldrich, 40,000 amu) and $\mathrm{Pd}\left(\mathrm{NO}_{3}\right)_{2} \cdot 2\left(\mathrm{H}_{2} \mathrm{O}\right)\left(5 \cdot 10^{-4} \mathrm{M}\right.$ Pd atoms; Sigma Aldrich, $40 \%$ wt. Pd basis) was heated to $368 \mathrm{~K}$ while stirring with a magnetic stir bar (310 rpm) in ambient air (one- 
neck flask, $0.1 \mathrm{~L}$, reflux condenser) for $0.4 \mathrm{~h}$. Spectral features of the Pd precursors were not detected via UV-visible spectroscopy of the colloidal suspension, thus indicating their complete reduction.

\subsubsection{Preparation of PVP protected Pd clusters $(2.3 \mathrm{~nm})$}

A solution of $\mathrm{Pd}\left(\mathrm{NO}_{3}\right)_{2} \cdot 2\left(\mathrm{H}_{2} \mathrm{O}\right)\left(5 \cdot 10^{-4} \mathrm{M}\right.$ Pd atoms; Sigma Aldrich, $40 \%$ wt. Pd basis $)$ in 1:1 by volume EtOH (Sigma Aldrich, $>99.5 \%$ ) and deionized $\mathrm{H}_{2} \mathrm{O}$ (conductivity $\sim 0.06 \mu \mathrm{S} \mathrm{cm}^{-1}$ ) was added to an equivalent volume of a monometallic $1.8 \mathrm{~nm}$ Pd cluster suspension $\left(5 \cdot 10^{-4} \mathrm{M} \mathrm{Pd}\right.$ atoms, prepared as described in Section 2.1.1). The resulting mixture was stirred (310 rpm) in ambient air (one-neck flask, $0.1 \mathrm{~L}$, reflux condenser) at $368 \mathrm{~K}$ for $0.33 \mathrm{~h}$. Spectral features of the $\mathrm{Pd}$ precursors were not detected via UV-visible spectroscopy of the colloidal suspension, thus indicating their complete reduction.

\subsubsection{Preparation of PVP protected PtPd clusters}

$\mathrm{H}_{2} \mathrm{PtCl}_{6}\left(5 \cdot 10^{-4} \mathrm{M} \mathrm{Pt}\right.$ atoms, Sigma Aldrich, $8 \%$ wt. in $\left.\mathrm{H}_{2} \mathrm{O}\right)$ in a mixture of $1: 1$ by volume EtOH (Sigma Aldrich, $>99.5 \%$ ) and deionized $\mathrm{H}_{2} \mathrm{O}$ (conductivity $\sim 0.06 \mu \mathrm{S} \mathrm{cm}^{-1}$ ) was added to an equivalent volume of the monometallic $1.8 \mathrm{~nm}$ Pd cluster suspension $\left(5 \cdot 10^{-4} \mathrm{M} \mathrm{Pd}\right.$ atoms, prepared as described in Section 2.1.1) and stirred (310 rpm) in ambient air (one-neck flask, 0.1 $\mathrm{L}$, reflux condenser) at $363 \mathrm{~K}$ for $0.33 \mathrm{~h}$ to form $\mathrm{Pt}_{50} \mathrm{Pd}_{50}$ clusters. Bimetallic PtPd clusters of different atomic compositions were synthesized by modifying the volumetric ratio of the Pd seed particle suspension and the Pt precursor solution. Spectral features of the Pt precursors were not detected via UV-visible spectroscopy of the colloidal suspension, thus indicating their complete reduction.

\subsubsection{Preparation of PVP protected Pt and AuPt clusters}

Pt and AuPt clusters were prepared by previously reported methods [14]. Briefly, Pt clusters were synthesized by heating a stirred solution of $\mathrm{H}_{2} \mathrm{PtCl}_{6}\left(5 \cdot 10^{-4} \mathrm{M} \mathrm{Pt}\right.$ atoms $)$ and $\mathrm{PVP}(0.02 \mathrm{M}$, monomeric unit basis) in ethylene glycol (EG) to $423 \mathrm{~K}$ in ambient air (one-neck flask, $0.1 \mathrm{~L}$, reflux condenser) for $0.33 \mathrm{~h}$. AuPt clusters were prepared by adding a solution of $\mathrm{HAuCl}_{4} \cdot 3\left(\mathrm{H}_{2} \mathrm{O}\right)\left(5 \cdot 10^{-4} \mathrm{M} \mathrm{Au}\right.$ atoms, in EG) at a rate of $16.7 \mathrm{nmol} \mathrm{Au} \mathrm{s}{ }^{-1}$ to a suspension of $\mathrm{Pt}$ clusters $\left(5 \cdot 10^{-4} \mathrm{M}\right.$ Pt atoms) maintained at $423 \mathrm{~K}$ (two-neck flask, $0.1 \mathrm{~L}$, reflux condenser). The resulting mixture was maintained at $423 \mathrm{~K}$ while stirring for $0.5 \mathrm{~h}$.

\subsubsection{Preparation of polyvinylalcohol (PVA) protected Pd and AuPd clusters}

$\mathrm{Pd}$ and AuPd clusters were prepared by previously reported methods [15]. Briefly, Pd clusters were synthesized by heating a stirred solution of $\mathrm{Pd}\left(\mathrm{NO}_{3}\right)_{2} \cdot 2\left(\mathrm{H}_{2} \mathrm{O}\right)\left(5 \cdot 10^{-4} \mathrm{M} \mathrm{Pd}\right.$ atoms $)$ and PVA (0.02 M, monomeric unit basis) in $1: 1$ by volume $\mathrm{EtOH}$ and deionized $\mathrm{H}_{2} \mathrm{O}$ to $368 \mathrm{~K}$ in ambient air (one-neck flask, $0.1 \mathrm{~L}$, reflux condenser) for $0.5 \mathrm{~h}$. AuPd clusters were prepared by adding a solution of $\mathrm{HAuCl}_{4} \cdot 3\left(\mathrm{H}_{2} \mathrm{O}\right)\left(5 \cdot 10^{-4} \mathrm{M} \mathrm{Au}\right.$ atoms, in $\left.1: 1 \mathrm{EtOH}: \mathrm{H}_{2} \mathrm{O}\right)$ to a suspension of $\mathrm{Pd}$ clusters $\left(5 \cdot 10^{-4} \mathrm{M} \mathrm{Pt}\right.$ atoms) and stirring the resulting mixture for $2 \mathrm{~h}$ at in air at ambient temperature (one-neck flask, 0.1 L). PVA was used as the protecting polymer in our initial bimetallic nanoparticle synthesis studies of AuPd clusters, however our more recent studies (i.e. AuPt and PtPd) use PVP because it typically offers greater colloidal stability over time and was more soluble in the solvents used.

\subsubsection{Dispersion of PVP protected clusters onto $\mathrm{SiO}_{2}$}


A Pd cluster suspension (52 $\mathrm{cm}^{3}$, prepared as described in Section 2.1.1) was added dropwise $\left(\sim 0.2 \mathrm{~cm}^{3} \mathrm{~s}^{-1}\right)$ to a suspension of fumed $\mathrm{SiO}_{2}(0.55 \mathrm{~g}$, Sigma Aldrich, $7 \mathrm{~nm}$ particle size, 395

$\mathrm{m}^{2} \mathrm{~g}^{-1}$ surface $)$ in deionized $\mathrm{H}_{2} \mathrm{O}\left(52 \mathrm{~cm}^{3}\right.$, conductivity $\sim 0.06 \mu \mathrm{S} \mathrm{cm}^{-1}$; Scheme 1.1c). The solids were collected by centrifugation $(133 \mathrm{~Hz}, 0.6 \mathrm{ks})$ after stirring the mixture for $1 \mathrm{~h}$ at ambient temperature. Complete deposition of clusters onto $\mathrm{SiO}_{2}$ was confirmed by the absence of absorbance in the UV-visible spectra of the supernatant solutions. Supported clusters were washed two times in deionized $\mathrm{H}_{2} \mathrm{O}\left(52 \mathrm{~cm}^{3}\right.$, conductivity $\left.\sim 0.06 \mu \mathrm{S} \mathrm{cm}^{-1}, 1 \mathrm{~h}\right)$ and subsequently treated overnight in ambient air at $356 \mathrm{~K}$. These procedures resulted in catalysts with $0.50 \%$ wt. metal loadings. Similar procedures were used to disperse bimetallic PtPd clusters onto $\mathrm{SiO}_{2}$, resulting in metal loadings of $0.50 \%$ wt., $0.58 \%$ wt., and $0.70 \%$ wt. for $\mathrm{Pt}_{10} \mathrm{Pd}_{90}, \mathrm{Pt}_{25} \mathrm{Pd}_{75}$, and $\mathrm{Pt}_{50} \mathrm{Pd}_{50}$ samples, respectively.

\subsection{Nanoparticle characterization}

\subsubsection{Transmission electron microscopy (TEM)}

A JEOL $1200 \mathrm{EX}$ microscope operated at $80 \mathrm{kV}$ was used to obtain bright-field transmission electron micrographs. TEM samples of colloidal Pd, AuPd, and PtPd clusters - synthesized in EtOH/ $\mathrm{H}_{2} \mathrm{O}$ mixtures - were prepared by depositing a drop of the cluster suspension in its synthesis medium onto holey-carbon $\mathrm{Cu}$ grids (Ted Pella Inc.) and allowing the solvent to evaporate in air at ambient temperature. TEM samples of colloidal Pt and AuPt samples synthesized in ethylene glycol, a less volatile solvent, were first re-dispersed in EtOH (methods reported previously [14]) in order to prepare grids without thermal treatments that could affect cluster composition and size. Clusters supported on $\mathrm{SiO}_{2}$ were first suspended in acetone before placing on $\mathrm{Cu}$ grids. A minimum of 200 clusters were analyzed per sample, and surface-averaged mean diameters were calculated from transmission electron micrographs using the equation:

$$
\langle d\rangle=\frac{\sum n_{i} d_{i}^{3}}{\sum n_{i} d_{i}^{2}}
$$

where $n_{i}$ is the number of particles with diameter $d_{i}$. The dispersity index $\left(D_{A}\right)$ was used to quantify particle size uniformity [21]:

$D_{A}=\frac{\sum n_{i} \sum\left(\pi d_{i}^{2}\right)^{2} n_{i}}{\left(\sum \pi d_{i}^{2} n_{i}\right)^{2}}$

\subsubsection{Ultraviolet-visible spectroscopy}

UV-visible spectra $(200-800 \mathrm{~nm})$ of cluster suspensions $\left(3 \mathrm{~cm}^{3}\right)$ in quartz cuvettes $\left(10 \mathrm{~cm}^{3}\right.$, Agilent) were collected using a two-beam spectrophotometer (Varian Cary 400 Bio) with a $5 \mathrm{~nm}$ $\mathrm{s}^{-1}$ scan rate. Spectra collected during synthesis were obtained by withdrawing $3 \mathrm{~cm}^{3}$ samples at specified time points using a disposable glass pipette.

\subsubsection{Elemental analysis of bimetallic clusters}

Energy dispersive X-ray spectroscopy (EDS) of particles from colloidal suspensions (EDS samples prepared as described in Section 2.2.1) were obtained using an FEI Titan microscope fitted with an FEI Super X-Quad windowless detector with silicon drift technology and operated at $200 \mathrm{kV}$. Atomic compositions were quantified using Cliff-Lorimer k-factors and Bruker Esprit 
software calibrated using mineral standards. High-angle annular dark field (HAADF) micrographs, collected concurrently to EDS X-rays, were acquired using a Fischione detector with an inner semi-angle $(\beta)$ of 50 milliradians and a convergence semi-angle (a) of 10 milliradians.

The diameters of bimetallic particles $\left(d_{M B M A}\right)$ were measured using ImageJ software and HAADF images. The volume-averaged mean atomic fraction of metal $\mathrm{M}\left(\left\langle x_{M}\right\rangle_{a t} ; \mathrm{M}=\mathrm{Au}, \mathrm{Pt}\right.$, or $\mathrm{Pd})$ in a sample of bimetallic clusters $(\mathrm{N}>100$ particles) was calculated by:

$\left\langle x_{M}\right\rangle_{a t .}=\frac{\sum x_{M, i} d_{M_{B} M_{A}, i}^{3}}{\sum d_{M_{B} M_{A}, i}^{3}}$

which accounts for larger particles containing a greater number of atoms than smaller particles.

2.3. Cleaning metal cluster surfaces and assessing the number of accessible surface atoms using $\mathrm{O}_{2}$ chemisorption

\subsubsection{Low-temperature treatment in $\mathrm{H}_{2}$ to remove the protective polymer}

$\mathrm{SiO}_{2}$-supported Pd particles (0.50\% wt.; prepared as described in Sections 2.1.1 and 2.1.6) and $\mathrm{SiO}_{2}$-supported bimetallic $\mathrm{Pt}_{10} \mathrm{Pd}_{90}, \mathrm{Pt}_{25} \mathrm{Pd}_{75}$, and $\mathrm{Pt}_{50} \mathrm{Pd}_{50}$ clusters $(0.50 \%$ wt., $0.58 \%$ wt., and $0.70 \%$ wt., respectively; prepared as described in Sections 2.1.2 and 2.1.6) were treated at $348 \mathrm{~K}\left(0.033 \mathrm{~K} \mathrm{~s}^{-1}\right)$ in flowing $\mathrm{H}_{2}$ (Praxair $99.999 \%, 0.25 \mathrm{~cm}^{3} \mathrm{~s}^{-1} \mathrm{~g}^{-1}$ ) for $1 \mathrm{~h}$ and subsequently treated at $423 \mathrm{~K}\left(0.033 \mathrm{~K} \mathrm{~s}^{-1}\right)$ for $1 \mathrm{~h}$ to remove the protective polymer (Scheme 1.1d). Chemisorbed $\mathrm{O}_{2}$ uptake was determined as described in Section 2.3.3 immediately after treatment.

\subsubsection{Low-temperature treatment in $\mathrm{EtOH} / \mathrm{H}_{2} \mathrm{O}$ and $\mathrm{H}_{2}$ to remove the protective polymer}

$\mathrm{SiO}_{2}$-supported Pd clusters (0.50\% wt.; prepared as described in Sections 2.1.1 and 2.1.6) were stirred in $50 \mathrm{~cm}^{3} \mathrm{~g}^{-1}$ of $1: 1$ by volume EtOH (Sigma Aldrich, >99.5\%) and deionized $\mathrm{H}_{2} \mathrm{O}$ (conductivity $\sim 0.06 \mu \mathrm{S} \mathrm{cm}^{-1}$ ) for $20 \mathrm{~h}$ at $351 \mathrm{~K}$ (in ambient air, one-neck flask, $0.1 \mathrm{~L}$, reflux condenser). Solids were collected by centrifugation $(133 \mathrm{~Hz}, 0.6 \mathrm{ks})$ and held in ambient air overnight at $353 \mathrm{~K}$ before being treated in flowing $\mathrm{H}_{2}$ (Praxair $99.999 \%, 0.25 \mathrm{~cm}^{3} \mathrm{~s}^{-1} \mathrm{~g}^{-1}$ ) at 423 $\mathrm{K}\left(0.033 \mathrm{~K} \mathrm{~s}^{-1}\right)$ for $1 \mathrm{~h}$ to remove the protective polymer (Scheme $\left.1.1 \mathrm{~d}\right)$. Chemisorbed $\mathrm{O}_{2}$ uptake was determined as described in Section 2.3.3 immediately after treatment.

\subsubsection{Chemisorbed $\mathrm{O}_{2}$ uptakes}

The number of accessible surface metal atoms was measured from catalyst $\mathrm{O}_{2}$ uptakes. A volumetric adsorption unit furnished with a Baratron gauge and a turbomolecular pumping system (Pfeiffer Vacuum; <1 Pa dynamic vacuum) was used to measure the amount of chemisorbed $\mathrm{O}_{2}$ for each sample. $\mathrm{SiO}_{2}$-supported $\mathrm{Pd}$ clusters $\left(0.50 \%\right.$ wt.) and $\mathrm{SiO}_{2}$-supported bimetallic $\mathrm{Pt}_{10} \mathrm{Pd}_{90}, \mathrm{Pt}_{25} \mathrm{Pd}_{75}$, and $\mathrm{Pt}_{50} \mathrm{Pd}_{50}$ clusters (0.50\% wt., $0.58 \%$ wt., and $0.70 \%$ wt., respectively), treated as described in Section 2.3.1 or 2.3.2, were evacuated at $423 \mathrm{~K}$ for $1 \mathrm{~h}$ and subsequently cooled to $298 \mathrm{~K}$. Isotherms were collected in a range from 0.013 to $101.3 \mathrm{kPa} \mathrm{O}_{2}$ (Praxair, $99.999 \%$ ), with $0.07 \mathrm{~h}$ allotted for equilibration with the gas phase at each pressure. Extrapolating to zero $\mathrm{O}_{2}$ pressure led to estimates of $\mathrm{O}_{2}$ uptakes. Cluster dispersions were calculated assuming one oxygen atom per metal surface atom [22]. The particle diameter was calculated from the chemisorption-derived dispersion $(D)$ using: 
$d=\frac{C}{D}$

where $C$ depends on the metal identity (determined by using the bulk density of the metals and assuming spherical clusters; $1.13 \mathrm{~nm}$ for Pt, $1.12 \mathrm{~nm}$ for Pd, and a weighted average, based on atomic composition, for bimetallic PtPd samples [23, 24]) and $d$ is diameter in nm. TEM-derived surface-averaged particle diameters similar to chemisorption-derived particle diameters indicate that the surfaces visible by TEM are fully accessible to probe molecules and free of synthetic detritus.

\section{Results and discussion}

\subsection{Synthesis and characterization}

Surface-averaged mean diameters (Eq. 1) of Pd clusters and bimetallic $\mathrm{Pt}_{33} \mathrm{Pd}_{67}, \mathrm{Pt}_{50} \mathrm{Pd}_{50}$, and $\mathrm{Pt}_{67} \mathrm{Pd}_{33}$ clusters were calculated from particle diameter measurements obtained by TEM (Fig. 1; Table 2). Mean PtPd cluster size increased monotonically with increasing Pt content, consistent with the reduction and deposition of solvated $\mathrm{Pt}$ cations onto the parent $\mathrm{Pd}$ seeds. Cluster uniformity was determined by calculating the dispersity index $\left(D_{A}\right)$ of PtPd samples using Equation 2 [21]. These $D_{A}$ values (1.13-1.20) were less than the value of 1.5 that IUPAC recommends to define monodispersity [21, 25]. Relative standard deviations (16-19\%), a more commonly reported but less rigorous measure of uniformity, are also included in Table 2, and similarly indicate that bimetallic PtPd clusters were narrowly distributed in size. Clusters with similar size distributions are typically obtained by colloidal methods using $\mathrm{NaBH}_{4}$ as a reductant and/or protecting agents with thiol groups [26, 27], which are difficult to remove from catalyst surfaces. The synthetic protocols reported here successfully avoid such heteroatoms without loss of size uniformity. 

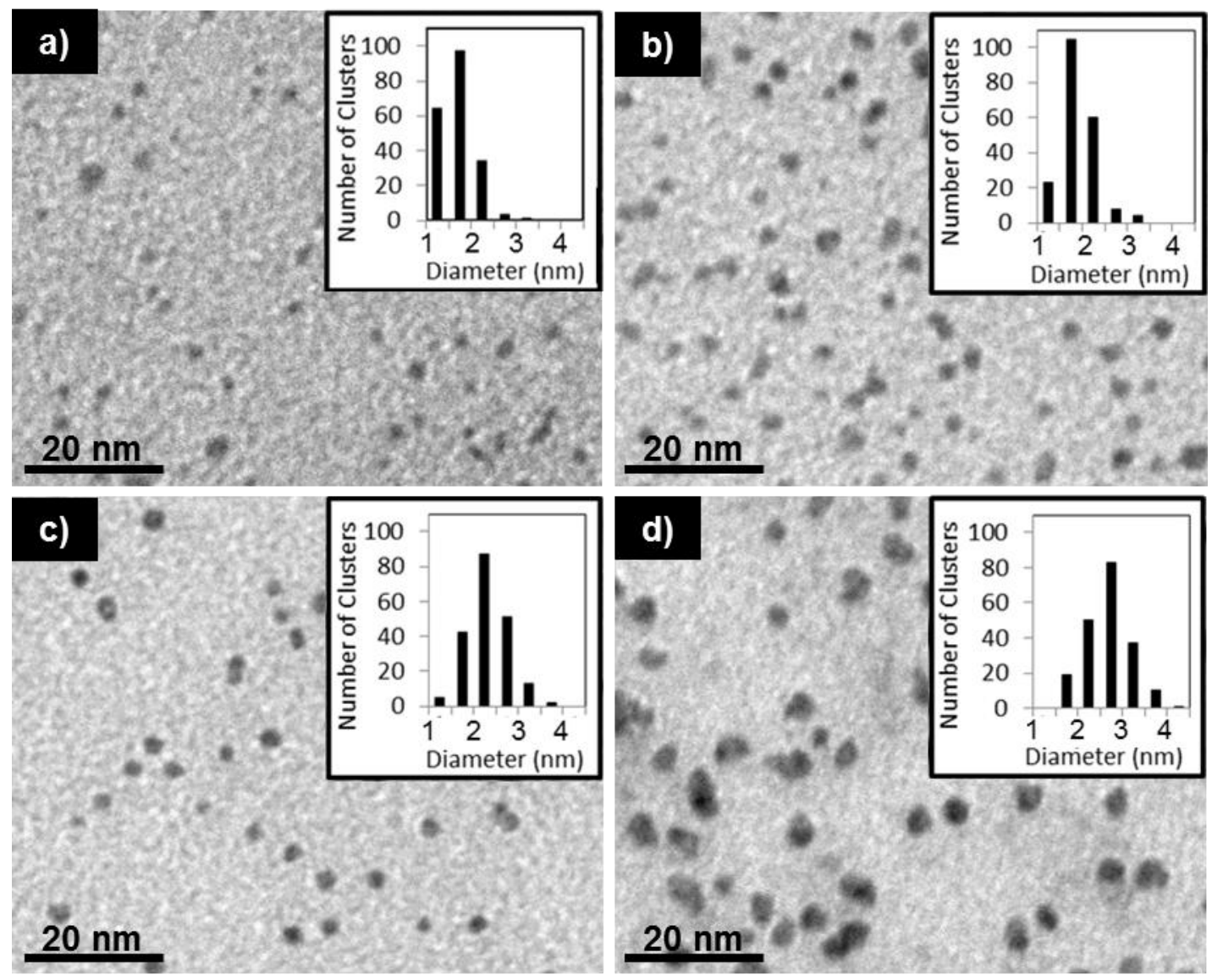

Figure 1. Cluster size distributions and transmission electron micrographs of a) $\mathrm{Pd}$, b) $\mathrm{Pt}_{33} \mathrm{Pd}_{67}$, c) $\mathrm{Pt}_{50} \mathrm{Pd}_{50}$, and d) $\mathrm{Pt}_{67} \mathrm{Pd}_{33}$ clusters.

Table 2. Surface-averaged mean diameters, expected bimetallic diameters, dispersity index values, and relative standard deviations

\begin{tabular}{ccccc} 
& \multicolumn{4}{c}{ Pt content (\% at.) } \\
\hline $\begin{array}{c}\text { TEM-derived surface-averaged } \\
\text { diameter, }(\mathrm{d}) \pm \text { STD. } \mathrm{N}^{-0.5} \\
(\mathrm{~nm}, \text { Eq. })\end{array}$ & 0 & 33 & 50 & 67 \\
$\begin{array}{c}\text { Expected diameter, } \mathrm{d}_{\text {PtPd }} \\
(\mathrm{nm}, \text { Eq. 5) }\end{array}$ & $1.8 \pm 0.02$ & $2.1 \pm 0.03$ & $2.5 \pm 0.03$ & $2.9 \pm 0.03$ \\
$\begin{array}{c}\text { Dispersity index, } D_{A} \\
(\text { Eq. 2) }\end{array}$ & -- & 2.2 & 2.5 & 2.8 \\
$\begin{array}{c}\text { Relative standard deviation } \\
(\%)\end{array}$ & 1.20 & 1.18 & 1.15 & 1.13 \\
\hline
\end{tabular}


Mean bimetallic cluster diameters $\left(\left\langle d_{M B M_{A}}\right\rangle\right.$; Table 2) predicted from Equation 5 were calculated for each composition using the measured surface-averaged mean diameter of the Pd seed clusters $\left(\left\langle d_{M_{A}}\right\rangle\right)$ :

$\left\langle d_{M_{B} M_{A}}\right\rangle=\left\langle d_{M_{A}}\right\rangle\left(1+\frac{V_{M_{B}}^{\prime} N_{M_{B}}}{V_{M_{A}}^{\prime} N_{M_{A}}}\right)^{1 / 3}$

where $V_{M x}^{\prime}$ is the atomic volume of metal $\mathrm{M}_{\mathrm{x}}$ and $N_{M x}$ is the number of atoms of metal $\mathrm{M}_{\mathrm{x}}$. Equation 5 assumes that solvated $\mathrm{Pt}\left(\mathrm{M}_{\mathrm{B}}\right)$ cations are reduced and deposited homogeneously across all $\operatorname{Pd}\left(\mathrm{M}_{\mathrm{A}}\right)$ seed clusters, thus precluding bimodal distributions of particle size. Such nonuniform size distributions would arise from the homogeneous nucleation and growth of monometallic Pt particles in solution or the nucleation of Pt on the surface of a fraction of seed clusters, known as heterogeneous nucleation; the former results in no cluster growth of the initial Pd seeds while the latter results in the growth of a fraction of seed particles that become larger at the expense of the remaining clusters. Figure 2 shows that measured $\left\langle d_{P t P d}\right\rangle$ values agreed well with those calculated from Equation 5, consistent with the absence of homogenous or heterogeneous nucleation of Pt during synthesis. No monometallic particles were detected from particle-by-particle elemental analysis using EDS ( $>100$ clusters per sample), thus confirming such conclusions. The mean atomic fractions of Pt and Pd (Eq. 3) within each bimetallic PtPd sample were consistent with the composition of the synthesis reagents used (Fig. 3a), consistent with the full recovery of all metal precursors in the solids.

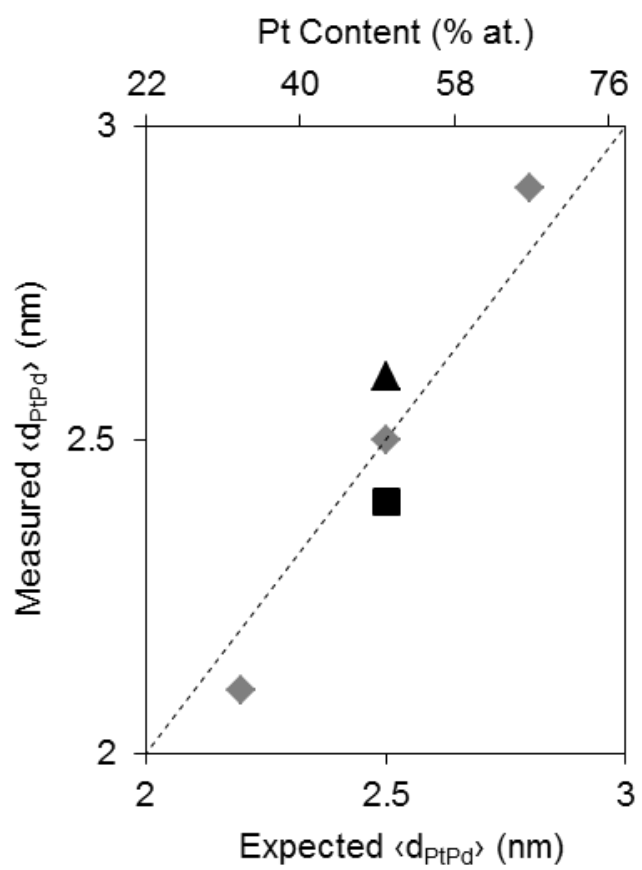

Figure 2. Parity plot of the measured (Eq. 2) and expected (Eq. 4) mean PtPd diameter $\left(\left\langle\mathrm{d}_{\mathrm{PtPd}}\right\rangle\right)$ of $\mathrm{Pt}_{33} \mathrm{Pd}_{67}, \mathrm{Pt}_{50} \mathrm{Pd}_{50}$, and $\mathrm{Pt}_{67} \mathrm{Pd}_{33}$ clusters synthesized at $363 \mathrm{~K}(\bullet)$, and of $\mathrm{Pt}_{50} \mathrm{Pd}_{50}$ clusters synthesized at ambient temperature (४) and $323 \mathrm{~K}(\boldsymbol{\square})$. 

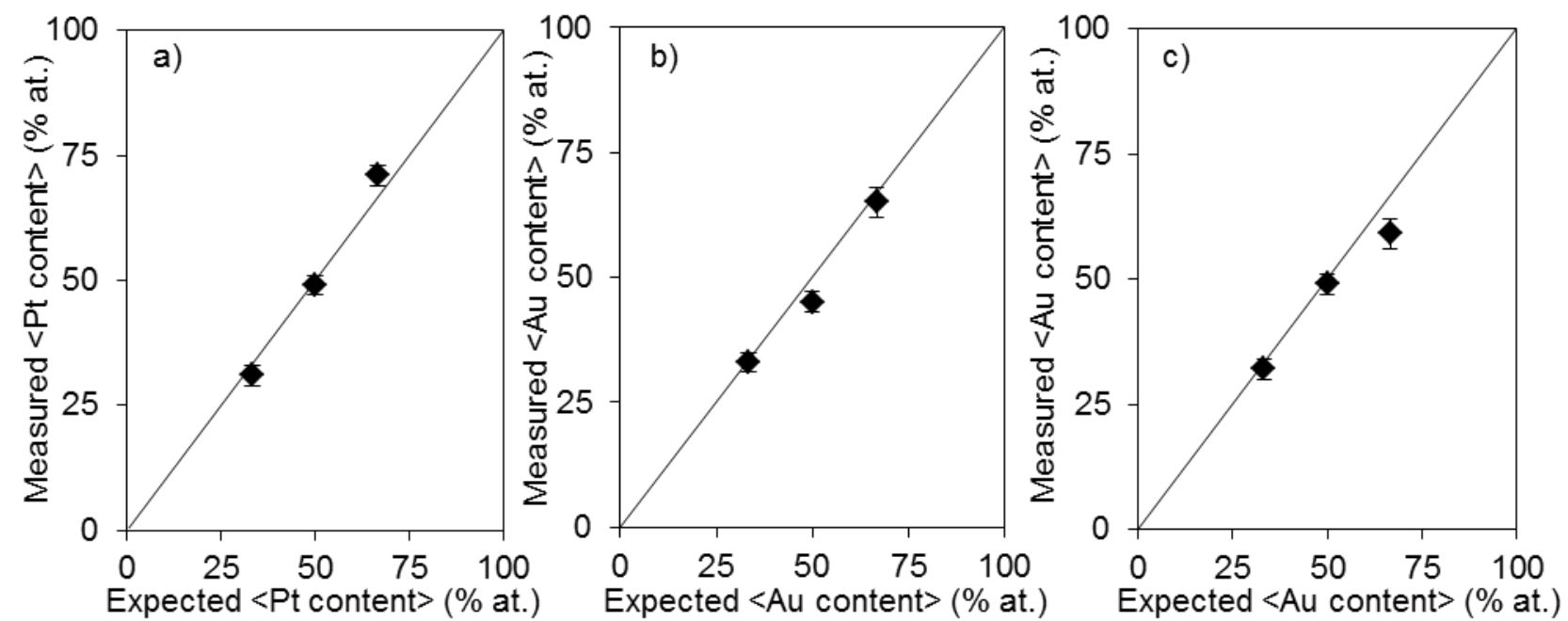

Figure 3. Parity plots of the mean $\mathrm{M}_{\mathrm{B}}$ content (\% at.; calculated from EDS data and Equation 3) and the expected $\mathrm{M}_{\mathrm{B}}$ content of a) PtPd, b) AuPd, and c) AuPt bimetallic samples. Over 100 clusters analyzed in each sample. Figures b) and c) reproduced from previously reported work [14] in order to generalize the results by comparisons among the three bimetallic compositions.

The disappearance of the $\mathrm{Pt}$ precursor band (266 nm [28]) during synthesis (ambient temperature, $1: 1$ by volume $\mathrm{EtOH} / \mathrm{H}_{2} \mathrm{O}$ solvent; Section 2.1.3) was monitored using UV-visible spectroscopy in order to confirm the full reduction of solvated Pt cations by the Pd seeds (Fig. 4a). The intensity of the Pt precursor band decreased (Fig. 4a) and the particle size increased (determined by TEM; Fig. 4b) with reaction time, consistent with the reduction and deposition of $\mathrm{Pt}$ cations as $\mathrm{Pt}^{0}$ onto the $\mathrm{Pd}$ seeds. Pt precursor features were no longer detected in the synthesis mixture after $46.5 \mathrm{~h}$, thus confirming the reduction of all solvated Pt cations. The UV-visible spectra of the clusters formed were similar to those of the initial Pd seed clusters (Fig. 5), as expected from the similar spectral properties of Pt and Pd clusters in the UV-visible range [29]. Higher synthesis temperatures led to the faster reduction of solvated Pt cations $(\sim 3 \mathrm{~h}$ at $323 \mathrm{~K}$, $\sim 0.33 \mathrm{~h}$ at $363 \mathrm{~K}$ ); the UV-visible spectra (Fig. 5) and the particle size distributions (Fig. 2) of the clusters formed at $298 \mathrm{~K}, 323 \mathrm{~K}$, and $363 \mathrm{~K}$ were similar. 

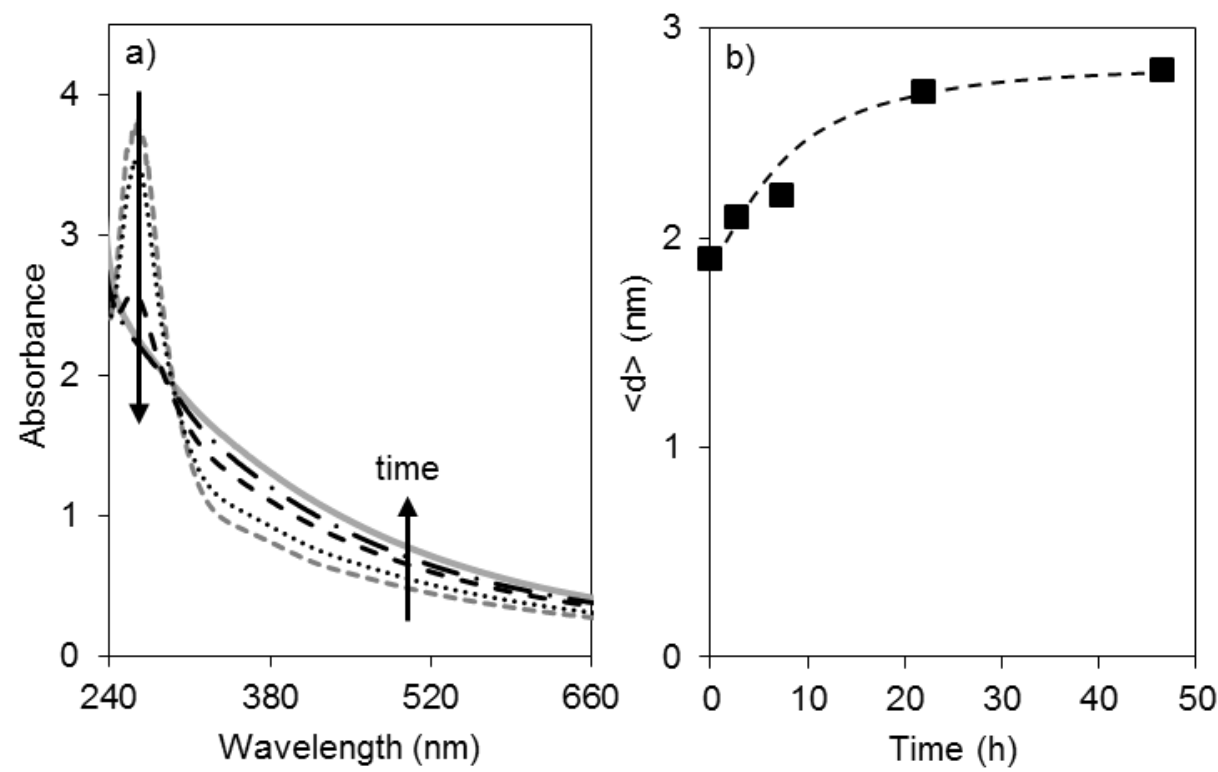

Figure 4. a) UV-visible spectra of $\mathrm{Pd}$ seed particles (_) and bimetallic $\mathrm{Pt}_{50} \mathrm{Pd}_{50}$ particles during synthesis at ambient temperature after $2.7 \mathrm{~h}\left({ }_{--}\right), 7.3 \mathrm{~h} \mathrm{(.....),} 22 \mathrm{~h} \mathrm{(.} \mathrm{-} \mathrm{),} \mathrm{and} 46.5 \mathrm{~h}$ (_. . . b) Surface-averaged particle diameters ( $\prec$ ¿; measured by TEM) with reaction time. Dashed curve is to guide the eye.

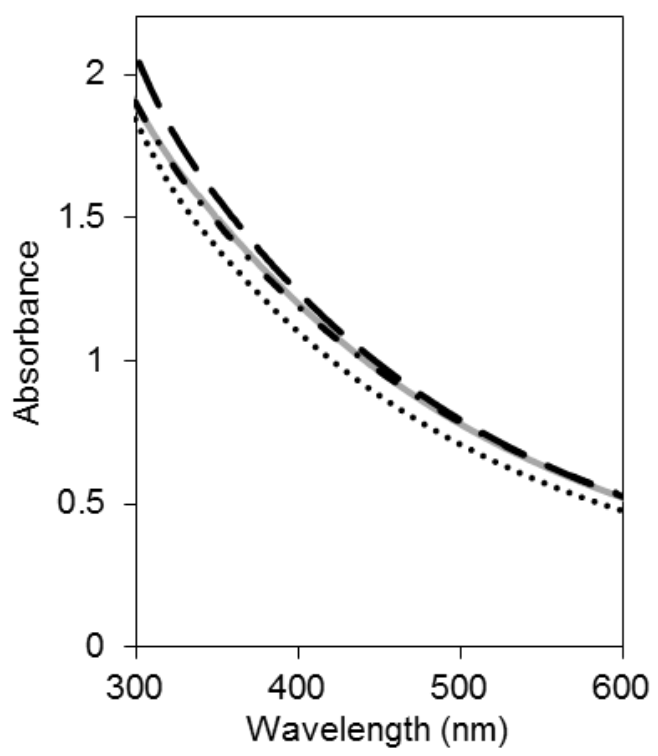

Figure 5. UV-visible spectra of Pd clusters ( $\left({ }_{\text {) }}\right.$ and bimetallic $\mathrm{Pt}_{50} \mathrm{Pd}_{50}$ clusters synthesized at

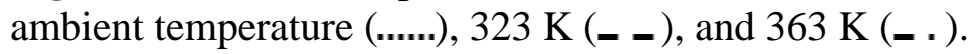

In contrast with solvated $\mathrm{Pt}$ cations, $\mathrm{Au}$ cations in contact with $\mathrm{Pd}$ seed clusters at ambient temperature reduced fully within $2 \mathrm{~h}$ (vs. $\sim 46.5 \mathrm{~h}$ for Pt; $\mathrm{EtOH} / \mathrm{H}_{2} \mathrm{O}$ mixtures used as solvent for both AuPd and PtPd synthesis [15]). Pd surface atoms reduce Pt cations more slowly than Au cations and thus form bimetallic PtPd clusters at lower rates than AuPd clusters. The relative kinetics of PtPd and AuPd formation reflects their different thermodynamic driving forces 
$\left(\Delta \mathrm{E}_{\text {redox }}^{\mathrm{o}}\right.$ ) for the kinetically relevant step of the GDR sequence, galvanic displacement (Scheme 1.1a), with the driving force for AuPd being greater than for PtPd systems $\left(\Delta \mathrm{E}_{\text {redox,AuPd }}^{\mathrm{o}}=0.74 \mathrm{~V}\right.$, $\Delta \mathrm{E}_{\text {redox }, \mathrm{PtPd}}^{\mathrm{o}}=0.24 \mathrm{~V}$; Table 1 ).

Unlike the Au-containing bimetallic systems reported previously (AuPt and AuPd) [14, 15], the alloyed metals discussed here (Pt and Pd) have similar UV-visible spectra (Fig. 5). We are thus unable to determine the predominant surface metal of the PtPd alloys, and cannot exclude the possibility that $\mathrm{Pt}$ cations deposit on activated Pd sites via electroless deposition (ED). Our previous studies lead us to suggest, however, that the thermodynamic driving force for galvanic displacement of Pd by Pt cations and the presence of a reducing agent to re-reduce oxidized $\mathrm{Pd}$ atoms would enhance the contributions from GDR pathways.

In addition to the magnitude of their $\Delta \mathrm{E}^{\mathrm{O}}$ redox values, the thermodynamic miscibility of the alloyed metals and the reduction potential of the solvent influence the need for specific protocols in order to prepare bimetallic clusters of uniform composition and size [14]. Enthalpically favored mixtures, such as AuPd and PtPd $\left(\Delta \mathrm{H}_{\mathrm{AuPd}}=-6.7 \mathrm{~kJ} \mathrm{~mol}^{-1}[18,30]\right.$ and $\Delta \mathrm{H}_{\mathrm{PtPd}}=-4.3 \mathrm{~kJ}$ $\left.\mathrm{mol}^{-1}[18,30]\right)$, can be synthesized at ambient temperatures [15]. In contrast, the formation of AuPt alloys $\left(\Delta \mathrm{H}_{\mathrm{AuPt}}=+2.3 \mathrm{~kJ} \mathrm{~mol}^{-1}\right.$ [20]) is enthalpically unfavorable and requires elevated synthesis temperatures $(423 \mathrm{~K}$ ) because the positive entropies of mixing become more influential in determining Gibbs free energies at higher temperatures [14]. Additionally, AuPt clusters were synthesized in a different solvent (ethylene glycol, EG) than that used for AuPd and PtPd synthesis $\left(1: 1\right.$ by volume EtOH: $\left.\mathrm{H}_{2} \mathrm{O}\right)$ because the Pt seed suspensions could not be stabilized in $\mathrm{EtOH} / \mathrm{H}_{2} \mathrm{O}$ mixtures. $\mathrm{EG}$, however, has a higher reduction potential than $\mathrm{EtOH}\left(\mathrm{E}_{\mathrm{EG}}^{\mathrm{o}}=-0.8 \mathrm{~V}\right.$ vs. $\mathrm{E}_{\mathrm{EtOH}}^{\mathrm{O}}=-0.197 \mathrm{~V}$ [17]); stronger reductants compete with $\mathrm{M}_{\mathrm{A}}$ surface atoms as reductants of $\mathrm{M}_{\mathrm{B}}$ cations, thus promoting the homogeneous or heterogeneous nucleation of clusters enriched in $\mathrm{M}_{B}$ (Scheme 1.2) that compromise cluster uniformity [14]. The synthesis of clusters in solvents with high reduction potentials, such as EG in the case of AuPt, therefore requires the slow drop-wise addition of the $M_{B}$ precursor solution in order to maintain low concentrations of solvated $M_{B}$ cations in solution, thereby inhibiting processes that lead to the nucleation of $\mathrm{M}_{\mathrm{B}}$-rich clusters.

\subsection{Steps and processes involved in the formation of bimetallic clusters}

Particle-by-particle elemental analysis using EDS is used next to probe the mechanistic features of bimetallic cluster formation via GDR processes. For parent clusters of element $\mathrm{M}_{\mathrm{A}}$ in a solution of solvated cations of element $M_{B}$, these processes require that $[14,15]$ :

(a) solvated $M_{B}$ cations be reduced exclusively by $M_{A}$ surface atoms (Scheme 1.1a),

(b) $\mathrm{M}_{\mathrm{A}}$ atoms brought into solution by galvanic displacement reactions in (a) be reduced by solvent molecules and deposited back onto particle surfaces (Scheme 1.1b), and

(c) $\mathrm{M}_{\mathrm{A}}$ atoms remain accessible at the growing cluster surface to (galvanically) reduce solvated $\mathrm{M}_{\mathrm{B}}$ cations, thus fulfilling requirement (a).

GDR growth therefore depends on the availability of $\mathrm{M}_{\mathrm{A}}$ surface atoms to reduce solvated $\mathrm{M}_{\mathrm{B}}$ cations as they reach cluster surfaces. When conditions (a)-(c) are met, the growth rate of a given particle, $i$, is proportional to the cluster surface area $\left(A_{i}\right)$ :

$\frac{d N_{i}}{d t}=A_{i} k$ 
where $k$ is the rate constant for galvanic displacement and $N_{i}$ is the number of atoms within the particle. The amount by which each cluster increases in diameter $\left(\Delta d_{i}\right)$ via addition of $\mathrm{M}_{\mathrm{B}}$ atoms can be derived from Equation 6 by expressing $A_{i}$ and $N_{i}$ as functions of the particle diameter $\left(d_{i}\right.$; derivation in Section S1, SI):

$\Delta d_{i}=2 V_{i}^{\prime} k t$

Here, $t$ is time and $V_{i}$ ' is the atomic volume of the elements in the particle. According to Equation 7, clusters that increase in diameter at a rate proportional to $A_{i}$ (Eq. 6) would increase in size by a value $\Delta d_{i}$ that that does not depend on the diameter of the parent $\mathrm{M}_{\mathrm{A}}$ cluster $[15,31$, 32]. Consequently, a suspension of $\mathrm{M}_{\mathrm{A}}$ seed particles of varying particle sizes in which each particle grows by the same thickness $(\Delta d)$ via addition of a second metal $\left(\mathrm{M}_{\mathrm{B}}\right)$ leads to bimetallic $\mathrm{M}_{\mathrm{B}} \mathrm{M}_{\mathrm{A}}$ clusters with a distribution of compositions for which the atomic fraction of $\mathrm{M}_{\mathrm{B}}$ decreases with increasing seed particle size (Scheme 2). This inverse relationship between the atomic fraction of $\mathrm{M}_{\mathrm{B}}$ and the seed particle diameter $\left(d_{M A}\right)$ is evident from a rearrangement of Equation 5 for a given particle, $i$ :

$\left(\frac{N_{M_{B}}}{N_{M_{A}}}\right)_{i}=\left[\left(1+\frac{\Delta d}{d_{M_{A}, i}}\right)^{3}-1\right] \frac{V^{\prime}{ }_{M_{A}}}{V^{\prime}{ }_{M_{B}}}$

where $d_{M_{B M A}}$ in Equation 5 has been replaced using Equation 9.

$d_{M_{B} M_{A}, i}=d_{M_{A}, i}+\Delta d$

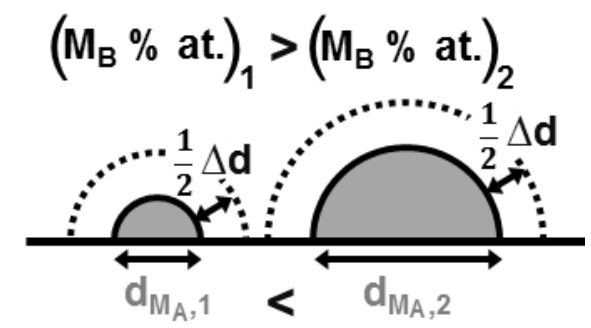

Scheme 2. $M_{A}$ seed particles of varying size that grow via galvanic displacement-reduction processes grow at a rate that is proportional to cluster surface area, and therefore increase in size by a thickness $(\Delta d)$ that is independent of the original seed particle diameter. Thus, the $\mathrm{M}_{\mathrm{B}}$ content ( $\%$ at.) of the resulting bimetallic particles is smaller for larger particles.

The compositional uniformity of bimetallic clusters formed from GDR processes is therefore determined by the breadth of the parent seed cluster size distribution. Next, we derive a relationship between the uniformity of the seed particle diameters and the compositional uniformity of GDR-derived bimetallic clusters using the coefficient of variation $(\mathrm{CV})$,

$C V=\frac{\sigma}{\mu}$ 
where $\sigma$ is the sample standard deviation and $\mu$ is the sample mean (of either seed diameters or bimetallic clusters compositions), as a measure uniformity. Probability density functions (PDFs) of varying $\mathrm{CV}$ value (0-0.4) were generated to represent seed particle diameters with log-normal size distributions [31] of varying uniformity, and were subsequently transformed into PDFs representing the compositions of the bimetallic particles formed via GDR processes; compositional uniformity was then quantified by calculating the CV values of the PDFs of the bimetallic compositions (derivation in Section S2, SI). Figure 6a shows how the CV of the bimetallic composition distributions decreases with decreasing $\mathrm{CV}$ of the seed particle diameters, thus demonstrating that sharper seed particle size distributions lead to concomitantly sharper compositional distributions.

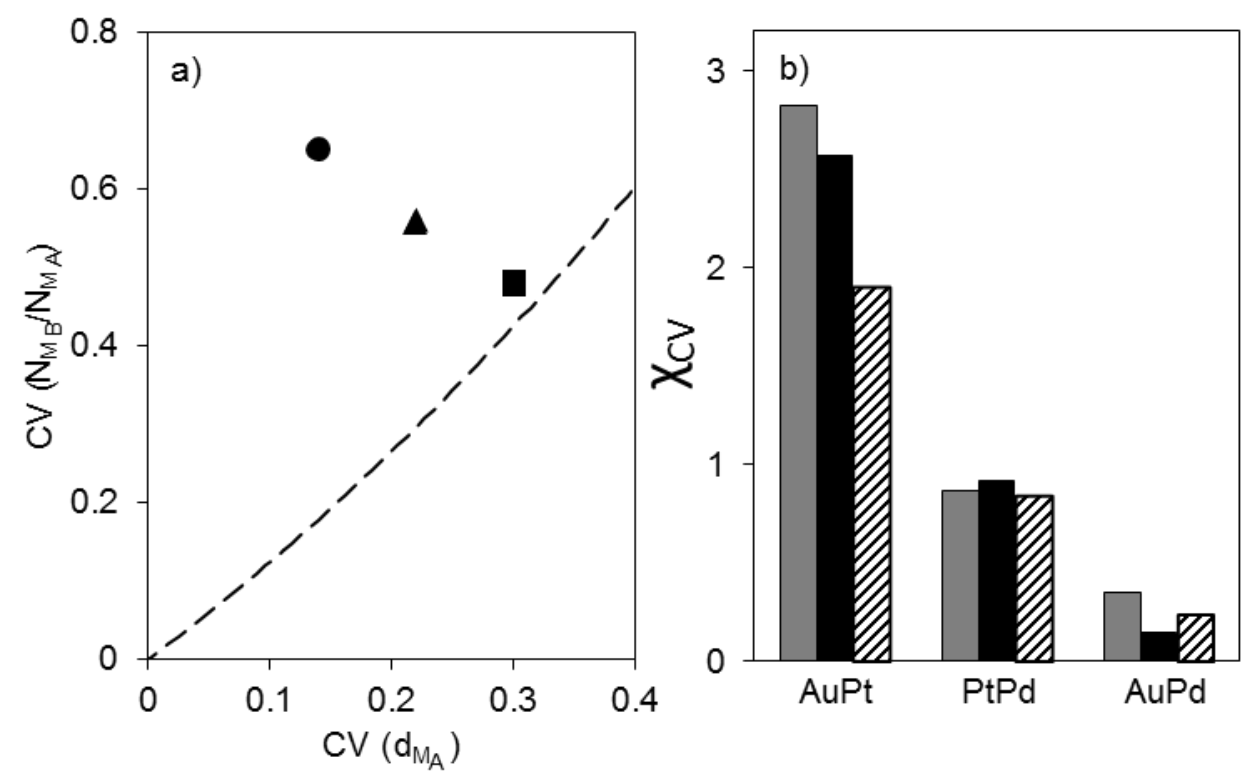

Figure 6. a) Expected relationship (_ - ) between the coefficient of variation (CV) of the parent seed cluster diameters, $\mathrm{d}_{\mathrm{MA}}$, and the $\mathrm{CV}$ of the resulting bimetallic cluster compositions, $\left(\mathrm{N}_{\mathrm{MB}} / \mathrm{N}_{\mathrm{MA}}\right)_{\text {at. }}$. Measured $\mathrm{CV}$ values for $\mathrm{Au}_{50} \mathrm{Pt}_{50}(\bullet), \mathrm{Pt}_{50} \mathrm{Pd}_{50}(\boldsymbol{\bullet})$, and $\mathrm{Au}_{50} \mathrm{Pd}_{50}(\boldsymbol{\square})$ clusters shown (determined from EDS data of bimetallic particle compositions and TEM of parent seed clusters). b) Deviation between the measured and expected compositional uniformity $\left(\chi_{\mathrm{CV}}, \mathrm{Eq}\right.$. 11) of AuPt, PtPd, and AuPd samples with $\mathrm{M}_{\mathrm{B}}$ contents of $33 \%$ at. (grey), $50 \%$ at. (black), and $67 \%$ at. (striped).

Any deviations from GDR pathways can be assessed from the curve in Figure 6a, derived for exclusive growth via GDR. Data obtained from EDS elemental analysis of bimetallic PtPd, AuPt, and AuPd clusters [14], together with transmission electron micrographs of Pt and Pd seed particle size distributions, are plotted in Figure 6a to assess whether measured compositional uniformity $\left(\mathrm{CV}_{\mathrm{EDS}}\right)$ deviates from that expected from GDR growth $\left(\mathrm{CV}_{\mathrm{GDR}}\right)$. Deviations from the curve in Figure $6 \mathrm{a}\left(\chi_{\mathrm{cv}}\right)$ are defined as:

$\chi_{C V}=\frac{C V_{E D S}-C V_{G D R}}{C V_{G D R}}$ 
AuPt clusters (an endothermic alloy) show the largest deviation from GDR behavior, while AuPd and PtPd clusters (exothermic alloys) show the smallest deviations (Fig. 6b), consistent with GDR reduction pathways being more prevalent during the synthesis of the latter two bimetallic systems. Such conclusions are consistent with PtPd and AuPd clusters that exhibit an inverse relation between $\%$ at. $\mathrm{M}_{\mathrm{B}}$ and seed cluster size (Fig. 7), as expected for GDR-derived clusters (Scheme 2); in contrast, the atomic fraction of AuPt clusters increases with increasing particle size. These data indicate that GDR requirements (a)-(c) prevail during AuPd and PtPd cluster synthesis but not during AuPt cluster synthesis, for which additional growth pathways must contribute significantly to particle growth.
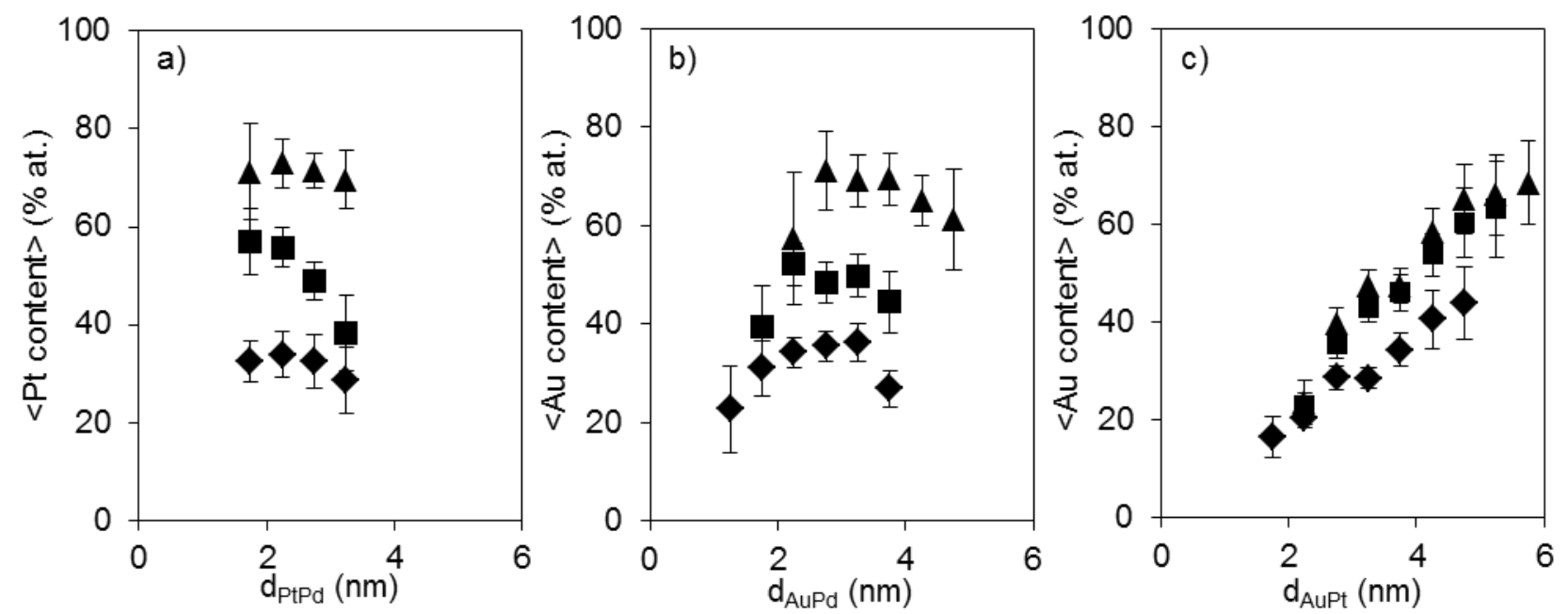

Figure 7. Changes in mean $\mathrm{M}_{\mathrm{B}}$ content (\% at.; calculated from EDS data) with increasing bimetallic particle diameter ( $\mathrm{d}_{\text {MBMA }}$, measured from HAADF images) for a) PtPd, b) AuPd, and c) AuPt particles. Data obtained for individual clusters and binned by particle size $(0.5 \mathrm{~nm}$ bin width). Bimetallic samples with nominal $\mathrm{M}_{\mathrm{B}}$ contents of $33 \%$ at. $(\bullet), 50 \%$ at. (ם), and $67 \%$ at. (A) presented. Over 100 clusters analyzed in each sample, with 74-98\% of clusters within 4 bins. Bins with fewer than three clusters excluded for clarity. Figures b) and c) reproduced from previously reported work [14] in order to generalize the results by comparisons among the three bimetallic compositions.

The formation mechanism can also be probed for these three bimetallic systems using HAADF images collected in tandem with particle-by-particle EDS analysis to measure both the diameters $\left(d_{M B M A, i}\right)$ and $\mathrm{M}_{\mathrm{A}}$ atomic fractions $\left(X_{M A, i}\right)$ of bimetallic clusters. These data are then used to calculate the size of the seed cluster from which each bimetallic cluster, $i$, formed and, in turn, the amount by which each seed particle increased in diameter $\left(\Delta d_{i}\right)$ using [14]:

$\Delta d_{i}=d_{M_{B} M_{A}, i}\left[1-\left(\frac{x_{M_{A}, i} V_{M_{A}}^{\prime}}{x_{M_{A}, i} V_{M_{A}}^{\prime}+x_{M_{B}, i} V_{M_{B}}^{\prime}}\right)^{1 / 3}\right]$

Here, $V_{M x}$ is the atomic volume of metal $\mathrm{M}_{\mathrm{x}}$ (derivation in Section S3, SI). Figures 8a-b show that the seed clusters of AuPd and PtPd particles grow by a value $\Delta d$ that is independent of seed particle size, as predicted for GDR growth. The expected $\Delta d$ value for each sample can be 
calculated from the TEM-derived size distribution of the parent seeds, onto which $\mathrm{M}_{\mathrm{B}}$ atoms are then distributed based on the surface area of each cluster, as dictated by GDR growth processes (derivation in Section S4, SI $[14,15])$. The expected $\Delta d$ values for AuPd and PtPd samples agree well with experimental values calculated from EDS and HAADF data (Figures 8a-b; dashed lines indicate expected $\Delta d$ values), consistent with the predominant involvement of GDR processes in their growth.
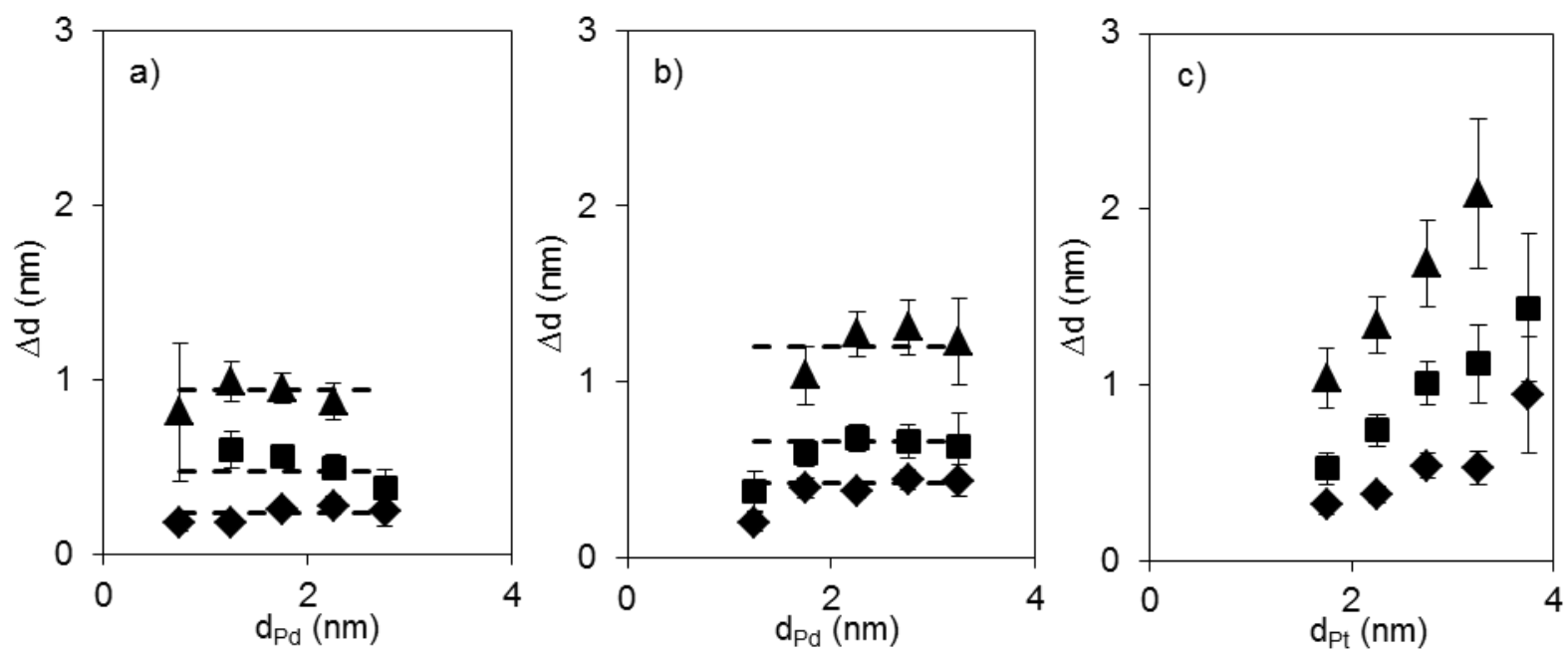

Figure 8. Effects of parent cluster diameter $\left(\mathrm{d}_{\mathrm{MA}}\right)$ on the growth of the parent seeds $(\Delta d$, calculated from EDS and HAADF data) via incorporation of $\mathrm{M}_{\mathrm{B}}$ atoms for a) PtPd, b) AuPd, and c) AuPt particles. Data obtained for individual clusters are binned by their initial seed particle diameters (0.5 $\mathrm{nm}$ bin width). Horizontal dashed lines (_ - ) in Figures a) and b) denote the expected $\Delta d$ value for PtPd and AuPd clusters, respectively, calculated using the initial seed particle size distribution (measured by TEM). Bimetallic samples with mean $\mathrm{M}_{\mathrm{B}}$ contents of 33

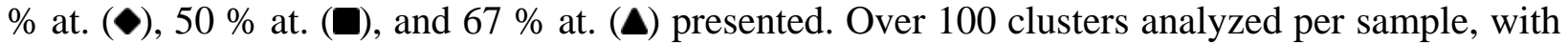
90-98\% of particles located in 4 bins. Bins with fewer than three clusters excluded for clarity. Figures b) and c) reproduced from previously reported work [14] in order to generalize the results by comparisons among the three bimetallic compositions.

In contrast, the Pt seed clusters in the AuPt system grow by a $\Delta d$ value that increased monotonically with the seed cluster diameter (Fig. 8c), consistent with bimetallic growth pathways other than GDR involved in their synthesis, discussed elsewhere [14]. Metal atoms within clusters of endothermic alloys, such as Au and Pt (mixing enthalpy, $\Delta \mathrm{H}_{\mathrm{AuPt}}=+2.3 \mathrm{~kJ} \mathrm{~mol}^{-}$ ${ }^{1}$ [20]), segregate in order to minimize the enthalpic penalties of mixing, thus driving the metal with the lowest surface energy, Au [33], to the surface (Scheme 3). The surface concentration of $\mathrm{Au}$ increases with cluster size because Au atom depletion from the cluster bulk is mitigated by their larger bulk reservoirs [34]. As the $\mathrm{Au}^{0}$ surface content of AuPt particles increases with particle size, they displace from the surface the $\mathrm{Pt}^{0}$ atoms required to reduce solvated $\mathrm{Au}$ cations via GDR, and instead promote the autocatalytic reduction of Au cations by solvent molecules on $\mathrm{Au}^{0}$ sites, a fast reaction [35,36]; this leads, in turn, to the preferential consumption of $\mathrm{Au}$ atoms by the surfaces of the larger clusters. Fast autocatalytic reduction on the larger clusters renders the uniform distribution of Au cations across all Pt seeds more challenging than for exothermic alloys (e.g., AuPd and PtPd), as reflected in the more stringent synthesis protocols required for 
the synthesis of AuPt clusters [14] than that for exothermic alloys that maintain $\mathrm{M}_{\mathrm{A}}$ atoms at cluster surfaces.

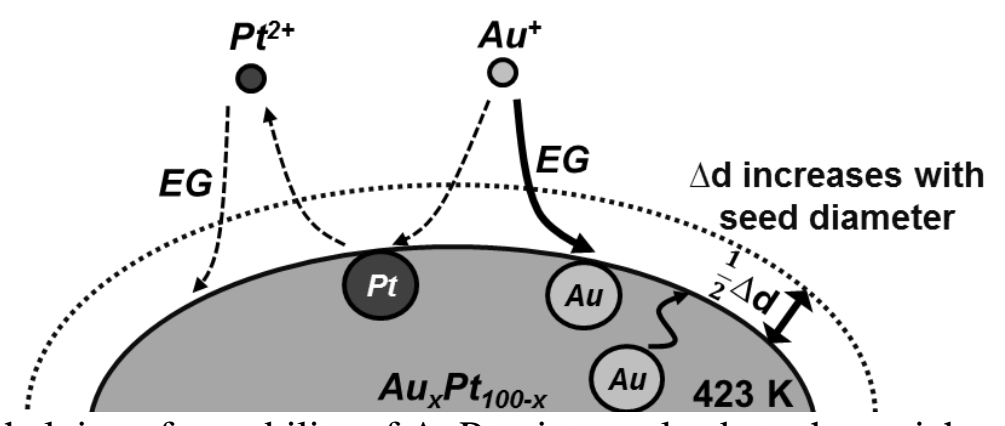

Scheme 3. The enthalpic unfavorability of AuPt mixtures leads to the enrichment $\mathrm{Au}^{0}$ at the surface of the larger AuPt particles during synthesis, thus impeding GDR processes while promoting the fast, autocatalytic reduction of solvated Au cations; larger Pt seed clusters therefore increase in diameter by a value $\Delta d$ that increases with seed cluster size. Scheme modified from previously reported work [14].

Our systematic study of the synthesis of AuPd, AuPt, and PtPd bimetallic systems has identified several key variables that influence the extent of metal mixing and the uniformity of the bimetallic clusters. Such features include the mixing enthalpy and relative reduction potentials of the alloyed metals, the solvent reduction potential, and the size uniformity of the parent seed clusters. Additionally, we have proposed several synthesis strategies to cope with those features that present challenges for the formation of bimetallic clusters uniform in composition and size. These synthesis protocols avoid the use of heteroatoms that ultimately compromise such uniformity because of the severe thermal and chemical treatments that become essential to remove synthetic debris from surfaces, a requirement of their ultimate use as catalysts.

\subsection{Achieving clean metal cluster surfaces using reductive treatments at mild conditions}

\subsection{1. $P V P$ removal from monometallic $P d$ cluster surfaces using $\mathrm{H}_{2}$}

Our previous studies of $\mathrm{SiO}_{2}$-supported monometallic $\mathrm{Pt}$ clusters showed that $\mathrm{PVP}$, the protective polymer used to prevent the agglomeration of Pt colloids, can be removed from cluster surfaces without causing particle growth by treating the catalyst in a gaseous $\left(\mathrm{H}_{2}\right)$ or liquid $(1: 1$ by volume EtOH: $\left.\mathrm{H}_{2} \mathrm{O}\right)$ reductant at low temperatures ( $\left.423 \mathrm{~K}\right)$ [14]. PVP binds to metal surfaces via the carbonyl oxygen of the pyrrolidone group [37]. The reductant detaches such species before reaching temperatures that lead to PVP densification and the formation of amorphous carbon. Such treatments avoid the consequences of $\mathrm{O}_{2}$ treatments requiring higher temperatures that often lead to the blockage of active surfaces by carbonaceous residues [37-40]. Here, we extend these protocols first to $\mathrm{SiO}_{2}$-supported $\mathrm{Pd}$ clusters protected by the presence of PVP in order to compare the monometallic systems, and then to bimetallic $\mathrm{SiO}_{2}$-supported PtPd clusters. The extent of polymer removal from treated cluster surfaces was probed by the titration of chemically accessible metal surface atoms with $\mathrm{O}_{2}$ to determine metal dispersions, which were then used to calculate surface-averaged particle diameters (Eq. 4) that can be compared with those measured from TEM micrographs. Particle diameters derived from chemisorption experiments that agree with those measured by TEM indicate that surfaces are free of synthetic 
residues. We expect that polymeric material remains on the support after these treatment techniques; if necessary, the removal of such polymer residues can be achieved by treatments at higher temperatures (> $423 \mathrm{~K}$ ) in oxidative environments [41].

The chemisorption-derived mean diameter of Pd clusters treated in flowing $\mathrm{H}_{2}$ using a twostep protocol previously shown to fully remove PVP from Pt cluster surfaces $(348 \mathrm{~K}$ for $1 \mathrm{~h}$, followed by $423 \mathrm{~K}$ for $1 \mathrm{~h}$; at a ramp rate of $0.033 \mathrm{~K} \mathrm{~s}^{-1}$; Section 2.3.1) was larger than the surface-averaged cluster diameter estimated from transmission electron micrographs (Fig. 9), indicating that residual polymeric material remained on metal surfaces after such treatments. Treated Pd clusters were also larger in size than untreated clusters (1.8 $\mathrm{nm}$ before treatment, 3.2 $\mathrm{nm}$ after treatment; determined by TEM). Since PVP binds to metal surfaces via carbonyl oxygen atoms [37-40] and the heat of oxygen adsorption is higher for Pd than $\mathrm{Pt}$ [42], the failure to fully remove PVP from Pd cluster surfaces using protocols suitable for Pt clusters appears to reflect the stronger binding energy of the polymer to Pd clusters. Incomplete polymer removal may also result from the smaller initial cluster size of the Pd clusters used here relative to the Pt clusters used previously (1.8 $\mathrm{nm}$ Pd clusters vs. $2.6 \mathrm{~nm}$ Pt clusters), which leads to a higher fraction of corner and edge surface atoms that bind adsorbates more strongly [43].

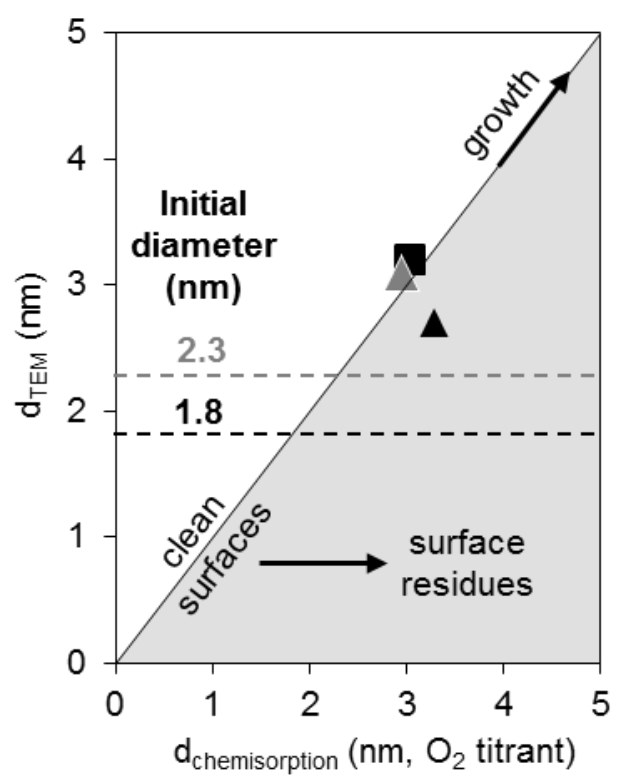

Figure 9. Parity plot of TEM-derived and chemisorption-derived particle diameters of $0.50 \%$ wt. $\mathrm{Pd} / \mathrm{SiO}_{2}$ after treatment in (triangle) flowing $\mathrm{H}_{2}\left(348 \mathrm{~K}\right.$ for $1 \mathrm{~h}$ and $423 \mathrm{~K}$ for $1 \mathrm{~h}, 0.033 \mathrm{~K} \mathrm{~s}^{-}$ ${ }^{1}$ ) or in (square) stirring 1:1 by volume $\mathrm{EtOH}: \mathrm{H}_{2} \mathrm{O}(351 \mathrm{~K}$ for $20 \mathrm{~h}$ ) followed by treatment in flowing $\mathrm{H}_{2}$ (423 K for $1 \mathrm{~h}$ ). Pd clusters with initial diameters of $1.8 \mathrm{~nm}$ (black) and $2.3 \mathrm{~nm}$ (grey) shown. Points on the parity line denote clean surfaces, with the initial untreated Pd particle size indicated by the horizontal dashed lines.

The metal-PVP bond on $1.8 \mathrm{~nm}$ Pd clusters may be weakened by populating the surface with metal atoms that bind oxygen more weakly, such as Pt (discussed later in Section 3.3.3), or by increasing the cluster size so as to reduce the fraction of under-coordinated surface atoms. Larger Pd clusters (2.3 nm; prepared as described in section 2.1.2) were treated using the protocols described in the preceding paragraph. In contrast to $1.8 \mathrm{~nm}$ Pd clusters, the surface-averaged particle diameter estimated from TEM images of the treated $2.3 \mathrm{~nm}$ clusters was similar to the 
diameter measured from chemisorption experiments (Fig. 9), consistent with clean and accessible metal surfaces. Larger particles with lower surface energies bind PVP more weakly, thus facilitating polymer removal and leading to clean metal surfaces. Despite these results, increasing the starting cluster diameter did not prevent particle growth during $\mathrm{H}_{2}$ treatment (from $2.3 \mathrm{~nm}$ to $3.1 \mathrm{~nm}$; determined by TEM). Pt clusters $(2.6 \mathrm{~nm})$ treated by identical protocols, however, did not increase in size [14], apparently because Pd clusters are more susceptible to sintering in $\mathrm{H}_{2}$ than $\mathrm{Pt}$ clusters [44].

\subsubsection{PVP removal from monometallic $\mathrm{Pd}$ cluster surfaces using $\mathrm{EtOH} / \mathrm{H}_{2} \mathrm{O}$ and $\mathrm{H}_{2}$}

The protocols developed to remove PVP from Pt cluster surfaces using $\mathrm{H}_{2}$ can be modified without compromising surface cleanliness or metal dispersion provided that clusters are treated in a reducing environment at mild temperatures for a sufficient period of time. Pt clusters treated in $\mathrm{H}_{2}$ at $423 \mathrm{~K}(1 \mathrm{~h})$ have clean surfaces but increase slightly in size; a preceding step at $348 \mathrm{~K}$ (1 $\mathrm{h}$ in $\mathrm{H}_{2}$ ) is required to fully remove the polymer slowly at lower temperatures and avoid local exotherms that lead to particle agglomeration because of fast exothermic polymer removal at such higher temperatures [14]. This additional treatment step in flowing $\mathrm{H}_{2}$ at $348 \mathrm{~K}$ can be substituted without compromising surface cleanliness or metal dispersion by instead treating Pt clusters in a reductive solvent $\left(1: 1\right.$ by volume EtOH: $\left.\mathrm{H}_{2} \mathrm{O}, 351 \mathrm{~K}, 20 \mathrm{~h}\right)$ before treatment at $423 \mathrm{~K}$ in $\mathrm{H}_{2}$, thus demonstrating the versatility of the reductive polymer removal approach [14].

The diameters measured from transmission electron micrographs of Pd clusters $(1.8 \mathrm{~nm})$ treated using the combined $\mathrm{EtOH} / \mathrm{H}_{2} \mathrm{O}$ and $\mathrm{H}_{2}$ protocol agreed well with those calculated from chemisorption experiments (Fig. 9), indicating that metal surfaces were free of chemisorbed material; clusters still increased in size (to $3.2 \mathrm{~nm}$; determined by TEM) as a result of this treatment. In contrast, the surfaces of $1.8 \mathrm{~nm}$ clusters treated using the protocols using $\mathrm{H}_{2}$ alone were not chemically accessible (Fig. 9). We therefore show here that protocols using $\mathrm{EtOH} / \mathrm{H}_{2} \mathrm{O}$ are more effective for removing PVP because of the greater contact time between the reductant and the metal surface $\left(20 \mathrm{~h}\right.$ in $\mathrm{EtOH} / \mathrm{H}_{2} \mathrm{O}$ at $351 \mathrm{~K}$ vs. $1 \mathrm{~h}$ in flowing $\mathrm{H}_{2}$ at $348 \mathrm{~K}$ ) and/or the ability of the solvent to dissolve and disperse the polymer away from cluster surfaces.

\subsubsection{PVP removal from bimetallic PtPd cluster surfaces using $\mathrm{H}_{2}$}

Pt clusters bind more weakly to oxygen-containing species, such as PVP, than Pd clusters [42], and are also more resistant to sintering in $\mathrm{H}_{2}$ [44]. Next, we investigate whether the presence of $\mathrm{Pt}$ atoms in $\mathrm{Pd}$ clusters will allow polymer removal without agglomeration. $\mathrm{Pt}_{10} \mathrm{Pd}_{90}$, $\mathrm{Pt}_{25} \mathrm{Pd}_{75}$, and $\mathrm{Pt}_{50} \mathrm{Pd}_{50}$ clusters were treated in flowing $\mathrm{H}_{2}(348 \mathrm{~K}$ for $1 \mathrm{~h}$, followed by $423 \mathrm{~K}$ for 1 $\mathrm{h}$; at a ramp rate of $0.033 \mathrm{~K} \mathrm{~s}^{-1}$; Section 2.3.1) and subsequently examined by TEM and titration with chemisorbed $\mathrm{O}_{2}$. The chemisorption-derived mean particle diameter of treated $\mathrm{Pt}_{10} \mathrm{Pd}_{90}$ clusters was larger than its TEM-derived surface-averaged mean diameter (Table 3), indicating that, as with monometallic Pd particles, surface residues remained; treated clusters were larger in size than untreated clusters ( $2 \mathrm{~nm}$ to $2.3 \mathrm{~nm}$; determined by TEM). In contrast, $\mathrm{Pt}_{50} \mathrm{Pd}_{50}$ clusters ( $2.5 \mathrm{~nm}$ diameter; determined by TEM) treated using identical protocols did not increase in size (Table 3); cluster diameters estimated from TEM images agreed well with those obtained from chemisorption experiments, indicating that clusters composed of $50 \%$ at. Pt were free of surface residues and resistant to coalescence during treatment. PtPd clusters with intermediate composition $\left(\mathrm{Pt}_{25} \mathrm{Pd}_{75}\right)$ exhibited intermediate cluster growth and surface cleanliness: clusters were slighter larger in size after $\mathrm{H}_{2}$ treatment $(2.4 \mathrm{~nm}$ to $2.6 \mathrm{~nm}$; determined by TEM) and had a chemisorption-derived diameter that was slightly larger than its TEM-derived diameter (Table 3). 
Table 3. Mean particle diameters of untreated and treated $\mathrm{PtPd} / \mathrm{SiO}_{2}$ clusters (measured by TEM or chemisorption)

\begin{tabular}{cccc}
$\begin{array}{c}\text { Pt content } \\
\text { (\% at.) }\end{array}$ & $\begin{array}{c}\mathrm{d}_{\text {TEM }} \\
\text { (untreated) }\end{array}$ & $\begin{array}{c}\mathrm{d}_{\text {TEM }} \\
\text { (treated in } \mathrm{H}_{2} \text { ) }\end{array}$ & $\begin{array}{c}\mathrm{d}_{\text {chpmion }} \\
10\end{array}$ \\
2 & 2.3 & 3.3 \\
25 & 2.4 & 2.6 & 2.7 \\
50 & 2.5 & 2.5 & 2.4 \\
\hline
\end{tabular}

The presence of Pt in PtPd clusters facilitates the removal of surface-bound PVP and renders clusters more resistant to sintering during treatment in $\mathrm{H}_{2}$. These data, however, cannot conclude whether the consequences of alloying reflect the increase in mean particle diameter, the increase in the amount of metal atoms that bind more weakly to PVP, or synergistic electronic effects of alloying Pt and Pd that decrease the binding energy of PVP to bimetallic surfaces. We expect that weaker metal-PVP bonds result from both the increase in particle size (as discussed in Section 3.3.1) and the presence of Pt at cluster surfaces. PtPd alloys are more resistant to poisoning by oxygen-binding surface species [45-47], however, suggesting that perhaps all three factors may influence PVP adsorption strength.

\section{Conclusions}

Using reagents composed of only $\mathrm{C}, \mathrm{O}, \mathrm{H}$, and $\mathrm{N}$, we have prepared colloidal PtPd clusters that are uniform in composition and size with chemically accessible metal surfaces. Clusters were formed via GDR processes - as confirmed by particle-by-particle elemental analysis using EDS and HAADF imaging - which lead to cluster growth $(\Delta d)$ that is independent of parent seed diameter. PtPd cluster formation was slower than the formation of AuPd clusters under identical conditions because of a lower thermodynamic driving force $\left(\Delta \mathrm{E}_{\text {redox }}^{\mathrm{o}}\right)$ for the reduction of $\mathrm{Pt}$ cations - relative to $\mathrm{Au}$ cations - by $\mathrm{Pd}^{0}$ surface atoms. PtPd and AuPd clusters can be synthesized at ambient temperature because of the enthalpic favorability of such metal combinations. In contrast, the positive mixing enthalpies of AuPt clusters require elevated synthesis temperatures $(423 \mathrm{~K})$ to promote the favorable entropic consequences of mixing that contribute more significantly to Gibbs free energies at higher temperatures.

Pd clusters were deposited onto $\mathrm{SiO}_{2}$ and subjected to reducing environments $\left(\mathrm{EtOH} / \mathrm{H}_{2} \mathrm{O}\right.$ or $\mathrm{H}_{2}$ ) at low temperatures (up to $423 \mathrm{~K}$ ) to remove the protecting polymer (PVP). Removing PVP from metal surfaces without compromising cluster dispersion was more challenging for Pd clusters relative to Pt clusters because of stronger metal-PVP bonds and the greater susceptibility of $\mathrm{Pd}$ clusters to sinter in $\mathrm{H}_{2}$. Increasing $\mathrm{Pd}$ cluster size and alloying with $\mathrm{Pt}$ were shown to facilitate polymer removal by weakening the metal-PVP bond.

Our work here, together with the conclusions drawn from the study of AuPd [15] and AuPt [14] bimetallic systems, illustrates the consequences of relative redox potentials, mixing enthalpies, solvent reduction potential, and parent seed size uniformity on the size and compositional uniformity of bimetallic clusters. We further demonstrate the versatility of GDR synthesis methods in adapting to unfavorable conditions that encourage bimodal particle 
distributions. The methods and predictive design principles developed for AuPd, AuPt, and PtPd systems can be generalized and applied to other bimetallic systems to synthesize the clean and uniform bimetallic catalysts that are essential for catalytic studies seeking to develop rigorous structure-function relationships of bimetallic surfaces.

\section{Acknowledgements}

The author would like to acknowledge the Achievement Rewards for College Scientists (ARCS) Fellowship and the National Science Foundation Graduate Research Fellowship (NSF GRF) for funding, the Electron Microscopy Lab (EML) at the University of California, Berkeley for TEM services, and the National Center for Electron Microscopy (NCEM), which is supported by the Office of Basic Energy Sciences of the U.S. Department of Energy under contract No. DEAC02-05CH11231, for access to EDS and HAADF facilities. This work was supported by the Office of Basic Energy Sciences, Chemical Sciences Division of the U.S. Department of Energy under contract No. DE-AC02-05CH11231. 


\section{References}

[1] J. Sinfelt, Bimetallic Catalysts, John Wiley \& Sons, Inc., New York, 1983.

[2] N. Toshima, Polymer-Protected Bimetallic Clusters. Preparation and Application to Catalysis, J. Macromol. Sci. Chem., 27 (1990) 1225-1238.

[3] C. Mihut, C. Descorme, D. Duprez, M.D. Amiridis, Kinetic and Spectroscopic Characterization of Cluster-Derived Supported Pt-Au Catalysts, J. Catal., 212 (2002) 125-135.

[4] Y.-H. Chin, E. Iglesia, Elementary Steps, the Role of Chemisorbed Oxygen, and the Effects of Cluster Size in Catalytic CH4-O2 Reactions on Palladium, J. Phys. Chem. C, 115 (2011) $17845-17855$.

[5] J. Lichtenberger, D. Lee, E. Iglesia, Catalytic oxidation of methanol on Pd metal and oxide clusters at near-ambient temperatures, Phys. Chem. Chem. Phys., 9 (2007) 4902-4906.

[6] B.M. Weiss, E. Iglesia, NO Oxidation Catalysis on Pt Clusters: Elementary Steps, Structural Requirements, and Synergistic Effects of NO2 Adsorption Sites, J. Phys. Chem. C, 113 (2009) 13331-13340.

[7] J. Rebelli, M. Detwiler, S. Ma, C.T. Williams, J.R. Monnier, Synthesis and characterization of $\mathrm{Au}-\mathrm{Pd} / \mathrm{SiO} 2$ bimetallic catalysts prepared by electroless deposition, J. Catal., 270 (2010) 224233.

[8] M.T. Schaal, A.C. Pickerell, C.T. Williams, J.R. Monnier, Characterization and evaluation of Ag-Pt/SiO2 catalysts prepared by electroless deposition, J. Catal., 254 (2008) 131-143.

[9] J. Rebelli, A.A. Rodriguez, S. Ma, C.T. Williams, J.R. Monnier, Preparation and characterization of silica-supported, group IB-Pd bimetallic catalysts prepared by electroless deposition methods, Catal. Today, 160 (2011) 170-178.

[10] K.D. Beard, J.W. Van Zee, J.R. Monnier, Preparation of carbon-supported Pt-Pd electrocatalysts with improved physical properties using electroless deposition methods, Appl. Catal., B, 88 (2009) 185-193.

[11] Y. Zhang, W. Diao, J.R. Monnier, C.T. Williams, Pd-Ag/SiO2 bimetallic catalysts prepared by galvanic displacement for selective hydrogenation of acetylene in excess ethylene, Catal. Sci. Technol., 5 (2015) 4123-4132.

[12] J. Zhang, Y. Mo, M.B. Vukmirovic, R. Klie, K. Sasaki, R.R. Adzic, Platinum Monolayer Electrocatalysts for $\mathrm{O} 2$ Reduction: Pt Monolayer on $\mathrm{Pd}(111)$ and on Carbon-Supported Pd Nanoparticles, J. Phys. Chem. B, 108 (2004) 10955-10964.

[13] N. Toshima, T. Yonezawa, Bimetallic nanoparticles-novel materials for chemical and physical applications, New J. Chem. , 22 (1998) 1179-1201.

[14] A.M. Landry, E. Iglesia, Synthesis of Bimetallic AuPt Clusters with Clean Surfaces via Sequential Displacement-Reduction Processes, Chem. Mater., 28 (2016) 5872-5886.

[15] S. Kunz, E. Iglesia, Mechanistic Evidence for Sequential Displacement-Reduction Routes in the Synthesis of Pd-Au Clusters with Uniform Size and Clean Surfaces, J. Phys. Chem. C, 118 (2014) 7468-7479.

[16] T. Yonezawa, N. Toshima, Mechanistic consideration of formation of polymer-protected nanoscopic bimetallic clusters, J. Chem. Soc., Faraday Trans., 91 (1995) 4111-4119.

[17] G. Milazzo, S. Caroli, R.D. Braun, Tables of standard electrode potentials, J. Electrochem. Soc., 125 (1978) 261C-261C.

[18] J.B. Darby Jr, The relative heats of formation of solid gold-palladium alloys, Acta Metall., 14 (1966) 265-270. 
[19] R. Watson, L. Swartzendruber, L. Bennett, Bonding effects in dilute transition-metal alloys, Phys. Rev. B, 24 (1981) 6211.

[20] L. Deng, W. Hu, H. Deng, S. Xiao, Surface Segregation and Structural Features of Bimetallic Au-Pt Nanoparticles, J. Phys. Chem. C, 114 (2010) 11026-11032.

[21] R. Gilbert, M. Hess, A. Jenkins, R. Jones, P. Kratochvil, R. Stepto, Dispersity in polymer science, Pure Appl. Chem, 81 (2009) 351-353.

[22] J.E. Benson, M. Boudart, Hydrogen-oxygen titration method for the measurement of supported platinum surface areas, J. Catal., 4 (1965) 704-710.

[23] M. Choi, Z. Wu, E. Iglesia, Mercaptosilane-Assisted Synthesis of Metal Clusters within Zeolites and Catalytic Consequences of Encapsulation, J. Am. Chem. Soc., 132 (2010) 91299137.

[24] M. Boudart, G. Djega-Mariadassou, Kinetics of Heterogeneous Catalysis, Princeton University Press, Princeton, NJ, (1984).

[25] K. Singh, J. Rouquerol, G. Bergeret, P. Gallezot, M. Vaarkamp, D. Koningsberger, A. Datye, J. Niemantsverdriet, T. Butz, G. Engelhardt, Characterization of Solid Catalysts: Sections 3.1. 1-3.1. 3, Handbook of Heterogeneous Catalysis, 427-582.

[26] H. Lang, S. Maldonado, K.J. Stevenson, B.D. Chandler, Synthesis and Characterization of Dendrimer Templated Supported Bimetallic Pt-Au Nanoparticles, J. Am. Chem. Soc., 126 (2004) 12949-12956.

[27] M.-L. Wu, D.-H. Chen, T.-C. Huang, Preparation of Au/Pt Bimetallic Nanoparticles in Water-in-Oil Microemulsions, Chem. Mater., 13 (2001) 599-606.

[28] D.L. Swihart, W.R. Mason, Electronic spectra of octahedral platinum (IV) complexes, Inorganic Chemistry, 9 (1970) 1749-1757.

[29] M. Vollmer, U. Kreibig, Optical properties of metal clusters, Springer Ser. Mat. Sci, 25 (1995).

[30] L.H. Bennett, R.E. Watson, A database for enthalpies of formation of binary transition metal alloys, Calphad, 5 (1981) 19-23.

[31] J.G. Brodnyan, Emulsion particle size III. Particle size distributions determined by various growth mechanisms, J. Colloid Sci., 15 (1960) 573-577.

[32] F. Goodrich, Nucleation rates and the kinetics of particle growth. I. The pure birth process, Proc. R. Soc. London, A, 1964, pp. 155-166.

[33] A.R. Miedema, The electronegativity parameter for transition metals: Heat of formation and charge transfer in alloys, J. Less-Common Met., 32 (1973) 117-136.

[34] F.L. Williams, D. Nason, Binary alloy surface compositions from bulk alloy thermodynamic data, Surf. Sci., 45 (1974) 377-408.

[35] C.H. Gammons, Y. Yu, A.E. Williams-Jones, The disproportionation of gold(I) chloride complexes at 25 to $200^{\circ} \mathrm{C}$, Geochim. Cosmochim. Acta, 61 (1997) 1971-1983.

[36] M.A. Watzky, R.G. Finke, Transition Metal Nanocluster Formation Kinetic and Mechanistic Studies. A New Mechanism When Hydrogen Is the Reductant: Slow, Continuous Nucleation and Fast Autocatalytic Surface Growth, J. Am. Chem. Soc., 119 (1997) 1038210400.

[37] Y. Borodko, S.E. Habas, M. Koebel, P. Yang, H. Frei, G.A. Somorjai, Probing the Interaction of Poly(vinylpyrrolidone) with Platinum Nanocrystals by UV-Raman and FTIR, J. Phys. Chem. B, 110 (2006) 23052-23059. 
[38] Y. Borodko, S.M. Humphrey, T.D. Tilley, H. Frei, G.A. Somorjai, Charge-Transfer Interaction of Poly(vinylpyrrolidone) with Platinum and Rhodium Nanoparticles, J. Phys. Chem. C, 111 (2007) 6288-6295.

[39] Y. Borodko, H.S. Lee, S.H. Joo, Y. Zhang, G. Somorjai, Spectroscopic Study of the Thermal Degradation of PVP-Capped Rh and Pt Nanoparticles in H2 and O2 Environments, J. Phys. Chem. C, 114 (2009) 1117-1126.

[40] J.N. Kuhn, C.-K. Tsung, W. Huang, G.A. Somorjai, Effect of organic capping layers over monodisperse platinum nanoparticles upon activity for ethylene hydrogenation and carbon monoxide oxidation, J. Catal., 265 (2009) 209-215.

[41] H. Song, R.M. Rioux, J.D. Hoefelmeyer, R. Komor, K. Niesz, M. Grass, P. Yang, G.A. Somorjai, Hydrothermal Growth of Mesoporous SBA-15 Silica in the Presence of PVPStabilized Pt Nanoparticles: Synthesis, Characterization, and Catalytic Properties, J. Am. Chem. Soc., 128 (2006) 3027-3037.

[42] R. van Santen, M. Neurock, Molecular Heterogeneous Catalysis: A Mechanistic and Computational Approach, VCH, in, Wiley, Inc, 2006.

[43] R. Van Hardeveld, F. Hartog, The statistics of surface atoms and surface sites on metal crystals, Surf. Sci., 15 (1969) 189-230.

[44] S.E. Wanke, P.C. Flynn, The sintering of supported metal catalysts, Catal. Rev., 12 (1975) 93-135.

[45] W. Wang, Q. Huang, J. Liu, Z. Zou, Z. Li, H. Yang, One-step synthesis of carbon-supported Pd-Pt alloy electrocatalysts for methanol tolerant oxygen reduction, Electrochem. Commun., 10 (2008) 1396-1399.

[46] F. Kadirgan, S. Beyhan, T. Atilan, Preparation and characterization of nano-sized Pt-Pd/C catalysts and comparison of their electro-activity toward methanol and ethanol oxidation, Int. J. Hydrogen Energy, 34 (2009) 4312-4320.

[47] T. Lopes, E. Antolini, E.R. Gonzalez, Carbon supported Pt-Pd alloy as an ethanol tolerant oxygen reduction electrocatalyst for direct ethanol fuel cells, Int. J. Hydrogen Energy, 33 (2008) 5563-5570. 